\title{
Nonclassical Problem for Ultraparabolic Equation in Abstract Spaces
}

\author{
Gia Avalishvili $^{1}$ and Mariam Avalishvili \\ ${ }^{1}$ Faculty of Exact and Natural Sciences, Ivane Javakhishvili Tbilisi State University, 3 I. Tchavtchavadze Avenue, 0179 Tbilisi, Georgia \\ ${ }^{2}$ School of Informatics, Engineering and Mathematics, University of Georgia, 77a M. Kostava Street, 0175 Tbilisi, Georgia
}

Correspondence should be addressed to Gia Avalishvili; gavalish@yahoo.com

Received 6 March 2016; Accepted 24 April 2016

Academic Editor: Maria Alessandra Ragusa

Copyright (C) 2016 G. Avalishvili and M. Avalishvili. This is an open access article distributed under the Creative Commons Attribution License, which permits unrestricted use, distribution, and reproduction in any medium, provided the original work is properly cited.

\begin{abstract}
Nonclassical problem for ultraparabolic equation with nonlocal initial condition with respect to one time variable is studied in abstract Hilbert spaces. We define the space of square integrable vector-functions with values in Hilbert spaces corresponding to the variational formulation of the nonlocal problem for ultraparabolic equation and prove trace theorem, which allows one to interpret initial conditions of the nonlocal problem. We obtain suitable a priori estimates and prove the existence and uniqueness of solution of the nonclassical problem and continuous dependence upon the data of the solution to the nonlocal problem. We consider an application of the obtained abstract results to nonlocal problem for ultraparabolic partial differential equation with second-order elliptic operator and obtain well-posedness result in Sobolev spaces.
\end{abstract}

\section{Introduction}

Evolution equations with several time-like variables are encountered in various models of science and technology, particularly, in mathematical models of multiparameter Brownian motion [1], theory of boundary layers [2], mathematical models of diffusion of pollutants in water flows [3], transport theory [4], mathematical models of age structured biological population dynamics $[5,6]$, mathematical finance [7], mechanics, physics, and cosmology [8, 9]. In these models one of time variables is usual time and others might denote various quantities, for example, coordinates, temperature, and age or size of individuals of biological population.

Various mathematical problems are investigated for multitime evolution equations. Ultraparabolic equations with classical Cauchy conditions with respect to one time variable, problems with classical initial conditions with respect to all time variables, and classical or nonlocal boundary conditions with respect to space variables, inverse problems are investigated in spaces of classical smooth functions and in suitable spaces of generalized functions, and some papers are also devoted to numerical methods for ultraparabolic equations with classical initial conditions (see [10-26] and references given therein). Abstract rather general ultraparabolic equation was studied by Lions [27] in spaces of abstract vectorvalued distributions. Applying methods of the theory of semigroups ultraparabolic integrodifferential equation with classical initial conditions with respect to time variables in the spaces of Hölder continuous vector-functions with values in a Banach space was investigated by Lorenzi [28] and ultraparabolic equations arising in age structured population dynamics with integral condition with respect to one of time variables were studied by many researchers; see $[6,29,30]$ and their references.

Mathematical models of populations incorporating age structure [6] are given by ultraparabolic equations and include nonlocal integral condition with respect to age variable, that is, nonlocal initial condition with respect to the second time variable. Nonclassical problem with nonlocal initial condition for abstract first-order evolution equation was investigated by Krejn and Laptev [31] and in the case of parabolic equation was studied by Kerefov [32]. Various initial-boundary value problems with nonlocal initial conditions are generalizations of initial-boundary value problems 
with classical and periodical initial conditions and they can be also considered as problems of controllability by initial conditions. Later on, initial-boundary value problems with nonlocal initial conditions were investigated for various timedependent partial differential equations and systems (see [33-45] and references given therein). Nonlocal in time problems with discrete nonlocal initial conditions are used for investigation of radionuclides propagation in Stokes fluid and problems of predicting the state of a medium $[44,45]$.

In this paper, we consider nonclassical problem for ultraparabolic equation:

$$
\frac{\partial u}{\partial t_{1}}+\frac{\partial u}{\partial t_{2}}+A u=f, \quad\left(t_{1}, t_{2}\right) \in\left(0, T_{1}\right) \times\left(0, T_{2}\right)
$$

with the following classical and nonlocal initial conditions:

$$
\begin{aligned}
& u\left(0, t_{2}\right)=\varphi\left(t_{2}\right), \quad 0 \leq t_{2} \leq T_{2}, \\
& u\left(t_{1}, 0\right)=\alpha u\left(t_{1}, \xi\right)+\psi\left(t_{1}\right), \quad 0 \leq t_{1} \leq T_{1},
\end{aligned}
$$

where $\alpha \in \mathbf{R}, 0<\xi<T_{2}, \varphi, \psi$, and $f$ are given vectorfunctions from suitable spaces, and $A$ is a given linear continuous operator in corresponding abstract Hilbert spaces.

As far as the authors know ultraparabolic equations with nonlocal initial condition (1) in abstract spaces have not been investigated yet. Moreover, in the case of classical initial conditions, that is, for $\alpha=0$, the well-posedness results are obtained either in spaces of continuous functions or in spaces of distributions with respect to time variables, for which the trace operator on boundary of domain of time variables cannot be defined. So, there are no results on the existence and uniqueness of solution in the spaces with minimal regularity properties necessary to define the initial conditions (2). In the present paper we obtain wellposedness theorem for nonlocal problem (1), (2), which in the case of $\alpha=0$ also gives new existence, uniqueness, and continuous dependence result for classical problem for the abstract ultraparabolic equation.

The paper is organized as follows. In Section 2, we introduce some notations and give properties of spaces of square integrable vector-functions defined on two-dimensional domain with values in Hilbert spaces. We prove new trace theorem and formulas for integration by parts. In addition, we show characteristic properties of some spaces of vectorvalued functions, which are used to investigate the nonclassical problem for ultraparabolic equation. In Section 3, we give variational formulation of the problem (1), (2) and prove our main result on the existence and uniqueness of its solution. Finally, in Section 4, we consider an application of the obtained abstract result to nonlocal problem for ultraparabolic partial differential equation in Sobolev spaces.

\section{Preliminaries}

We denote by $L(X ; Y)$ the space of linear continuous operators from $X$ to $Y$, where $X$ and $Y$ are Banach spaces. Let $C([0, T] ; X)$ denote the space of continuous vector-functions on $[0, T]$ with values in $X$. We denote by $L^{2}(\Delta ; X)$ the space of measurable vector-functions $g: \Delta \rightarrow X$ such that $\|g\|_{X} \epsilon$ $L^{2}(\Delta)$ and the generalized derivatives of $g$ are denoted by $\partial g / \partial \tau_{i} \in D^{\prime}(\Delta ; X)=L(D(\Delta) ; X), i=1, \ldots, s, d g / d \tau_{1}=g^{\prime}$, for $s=1$ (cf. [46]), where $D(\Delta)$ is the space of infinitely differentiable functions with compact support in $\Delta$, where $\Delta \subset \mathbf{R}^{s}, s \in \mathbf{N}$ is a bounded domain.

Throughout this paper, we use $c$ to denote generic constants that are independent of the main parameters involved, but whose values may differ from line to line and may change even within a single chain of estimates.

Let $V$ and $H$ be separable Hilbert spaces, such that $V$ is dense in $H$ and continuously embedded in it. We identify space $H$ with its dual by using inner product $(\cdot, \cdot)$ in $H$ and hence $V \subset H \subset V^{\prime}$ with continuous and dense embeddings, where $V^{\prime}$ is the dual space of $V$ [46]. We denote by $\langle\cdot, \cdot\rangle$ the duality relation between $V^{\prime}$ and $V$. To investigate problem (1), (2) let us consider the following space of vector-valued functions on $\omega=\left(0, T_{1}\right) \times\left(0, T_{2}\right)$ :

$$
W=\left\{w \in L^{2}(\omega ; V) ; \frac{\partial w}{\partial t_{1}}, \frac{\partial w}{\partial t_{2}} \in L^{2}\left(\omega ; V^{\prime}\right)\right\}
$$

which is a Hilbert space equipped with the norm

$$
\begin{aligned}
& \|v\|_{W} \\
& \quad=\left(\|v\|_{L^{2}(\omega ; V)}^{2}+\left\|\frac{\partial w}{\partial t_{1}}\right\|_{L^{2}\left(\omega ; V^{\prime}\right)}^{2}+\left\|\frac{\partial w}{\partial t_{2}}\right\|_{L^{2}\left(\omega ; V^{\prime}\right)}^{2}\right)^{1 / 2} .
\end{aligned}
$$

In the following three theorems we give some properties of the space $W$.

Theorem 1. For each vector-function $w \in W$ and $0 \leq \tau_{1} \leq$ $T_{1}, 0 \leq \tau_{2} \leq T_{2}$, there exist traces $w\left(\tau_{1}, t_{2}\right)=\left(\operatorname{tr}_{t_{1}=\tau_{1}} w\right)\left(t_{2}\right)$ and $w\left(t_{1}, \tau_{2}\right)=\left(\operatorname{tr}_{t_{2}=\tau_{2}} w\right)\left(t_{1}\right)$, such that the trace operators $\operatorname{tr}_{t_{1}=\tau_{1}}: W \rightarrow L^{2}\left(0, T_{2} ; H\right)$ and $\operatorname{tr}_{t_{2}=\tau_{2}}: W \rightarrow L^{2}\left(0, T_{1} ; H\right)$ are continuous.

Proof. We can identify each vector-function $v$ from space $L^{2}(\omega ; V)$ with vector-function $\widetilde{v}$ from $L^{2}\left(0, T_{1} ; L^{2}\left(0, T_{2} ; V\right)\right)$. If $w \in W$, then $\partial w / \partial t_{1} \in L^{2}\left(\omega ; V^{\prime}\right)$, and since the linear combinations of products $\theta \eta, \theta \in D\left(0, T_{1}\right)$, and $\eta \in D\left(0, T_{2}\right)$ are dense in $D(\omega)$, we have $\widetilde{w} \in L^{2}\left(0, T_{1} ; L^{2}\left(0, T_{2} ; V\right)\right)$, d $\widetilde{w} / d t_{1}$ $\in L^{2}\left(0, T_{1} ; L^{2}\left(0, T_{2} ; V^{\prime}\right)\right)$. Note that $L^{2}\left(0, T_{2} ; V\right) \subset L^{2}\left(0, T_{2}\right.$; $H) \subset L^{2}\left(0, T_{2} ; V^{\prime}\right)$ with continuous and dense embeddings and $L^{2}\left(0, T_{2} ; V^{\prime}\right)=\left(L^{2}\left(0, T_{2} ; V\right)\right)^{\prime}$. Consequently, from embedding theorem $[46]$ we have that $\widetilde{w} \in C\left(\left[0, T_{1}\right] ; L^{2}\left(0, T_{2}\right.\right.$; $H))$ and $C\left(\left[0, T_{1}\right] ; L^{2}\left(0, T_{2} ; H\right)\right)$ is continuously embedded in

$$
\begin{aligned}
W_{1} & =\left\{w_{1} \in L^{2}\left(0, T_{1} ; L^{2}\left(0, T_{2} ; V\right)\right) ; \frac{d w}{d t_{1}}\right. \\
& \left.\in L^{2}\left(0, T_{1} ; L^{2}\left(0, T_{2} ; V^{\prime}\right)\right)\right\} ;
\end{aligned}
$$


that is,

$$
\begin{aligned}
& \left\|\widetilde{w}\left(\tau_{1}\right)\right\|_{L^{2}\left(0, T_{2} ; H\right)} \\
& \quad \leq c\left(\|\widetilde{w}\|_{L^{2}\left(0, T_{1} ; L^{2}\left(0, T_{2} ; V\right)\right)}^{2}+\left\|\frac{d \widetilde{w}}{d t_{1}}\right\|_{L^{2}\left(0, T_{1} ; L^{2}\left(0, T_{2} ; V^{\prime}\right)\right)}^{2}\right)^{1 / 2} \\
& \quad \leq c\|w\|_{W},
\end{aligned}
$$

for any $\tau_{1} \in\left[0, T_{1}\right]$. Hence, we can define the trace operator $w\left(\tau_{1}, t_{2}\right)=\left(\operatorname{tr}_{t_{1}=\tau_{1}} w\right)\left(t_{2}\right)=\widetilde{w}\left(\tau_{1}\right)$, which is a linear continuous operator from $W$ to $L^{2}\left(0, T_{2} ; H\right)$. Likewise, for each $0 \leq$ $\tau_{2} \leq T_{2}$ we obtain the existence of the linear continuous trace operator $w\left(t_{1}, \tau_{2}\right)=\left(\operatorname{tr}_{t_{2}=\tau_{2}} w\right)\left(t_{1}\right)$ from $W$ to $L^{2}\left(0, T_{1} ; H\right)$.

Theorem 2. For vector-functions $w, w_{*} \in W$ the following formulas for integration by parts are valid:

$$
\begin{aligned}
& \int_{0}^{T_{2}} \int_{0}^{T_{1}}\left\langle\frac{\partial w}{\partial t_{1}}, w_{*}\right\rangle d t_{1} d t_{2} \\
& \quad+\int_{0}^{T_{2}} \int_{0}^{T_{1}}\left\langle\frac{\partial w_{*}}{\partial t_{1}}, w\right\rangle d t_{1} d t_{2} \\
& \quad=\int_{0}^{T_{2}}\left(w\left(T_{1}, t_{2}\right), w_{*}\left(T_{1}, t_{2}\right)\right) d t_{2} \\
& \quad-\int_{0}^{T_{2}}\left(w\left(0, t_{2}\right), w_{*}\left(0, t_{2}\right)\right) d t_{2}, \\
& \int_{0}^{T_{1}} \quad \int_{0}^{T_{2}}\left\langle\frac{\partial w}{\partial t_{2}}, w_{*}\right\rangle d t_{2} d t_{1} \\
& \quad+\int_{0}^{T_{1}} \int_{0}^{T_{2}}\left\langle\frac{\partial w_{*}}{\partial t_{2}}, w\right\rangle d t_{2} d t_{1} \\
& \quad=\int_{0}^{T_{1}}\left(w\left(t_{1}, T_{2}\right), w_{*}\left(t_{1}, T_{2}\right)\right) d t_{1} \\
& \quad-\int_{0}^{T_{1}}\left(w\left(t_{1}, 0\right), w_{*}\left(t_{1}, 0\right)\right) d t_{1} .
\end{aligned}
$$

Proof. For $w, w_{*} \in W$ we consider the corresponding vectorfunctions $\widetilde{w}, \widetilde{w}_{*} \in W_{1}$ defined in the proof of Theorem 1 . For vector-functions $\widetilde{w}, \widetilde{w}_{*} \in W_{1}$ the following formula for integration by parts is valid [46]:

$$
\begin{gathered}
\int_{0}^{T_{1}}\left\langle\frac{d \widetilde{w}}{d t_{1}}, \widetilde{w}_{*}\right\rangle_{T_{2}} d t_{1}+\int_{0}^{T_{1}}\left\langle\frac{d \widetilde{w}_{*}}{d t_{1}}, \widetilde{w}\right\rangle_{T_{2}} d t_{1} \\
=\left(\widetilde{w}\left(T_{1}\right), \widetilde{w}_{*}\left(T_{1}\right)\right)_{T_{2}}-\left(\widetilde{w}(0), \widetilde{w}_{*}(0)\right)_{T_{2}},
\end{gathered}
$$

where $\langle\cdot, \cdot\rangle_{T_{2}}$ denotes the duality relation between $L^{2}\left(0, T_{2}\right.$; $\left.V^{\prime}\right)$ and $L^{2}\left(0, T_{2} ; V\right)$ and $(\cdot, \cdot)_{T_{2}}$ denotes the inner product in $L^{2}\left(0, T_{2} ; H\right)$. The latter formula is equivalent to the first formula of the theorem. Likewise we obtain the second formula.
Theorem 3. For vector-functions $w \in W$ and $v \in V$ the following equalities are valid:

$$
\begin{aligned}
& \left\langle\frac{\partial w}{\partial t_{1}}, v\right\rangle=\frac{\partial}{\partial t_{1}}(w, v), \\
& \left\langle\frac{\partial w}{\partial t_{2}}, v\right\rangle=\frac{\partial}{\partial t_{2}}(w, v)
\end{aligned}
$$

in $D^{\prime}(\omega)$.

Proof. Applying Theorem 2 for $w \in W$ and $w_{*}=\phi v, \phi \in$ $D(\omega), v \in V$, we obtain

$$
\begin{gathered}
\int_{0}^{T_{2}} \int_{0}^{T_{1}}\left\langle\frac{\partial w}{\partial t_{i}}, v\right\rangle \phi\left(t_{1}, t_{2}\right) d t_{1} d t_{2} \\
=-\int_{0}^{T_{2}} \int_{0}^{T_{1}}\langle w, v\rangle \frac{\partial \phi}{\partial t_{i}} d t_{1} d t_{2}=0
\end{gathered}
$$

for $i=1,2$. Since $w\left(t_{1}, t_{2}\right) \in V,\left(t_{1}, t_{2}\right) \in \omega$, we have $\langle w, v\rangle=$ $(w, v)$, which proves the required equalities.

Let $A \in L\left(V ; V^{\prime}\right)$ be a self-adjoint coercive operator, which means that the bilinear form $a(v, w)=\langle A v, w\rangle$ satisfies the conditions

$$
\begin{gathered}
|a(v, w)| \leq c_{a}\|v\|_{V}\|w\|_{V}, \\
a(v, w)=a(w, v), \\
a(v, v) \geq \widehat{c}_{a}\|v\|_{V}^{2},
\end{gathered}
$$

$\forall v, w \in V$,

where $c_{a}, \widehat{c}_{a}=$ const $>0$. We also suppose that there exists a system of eigenvectors $\left\{v_{k}\right\}_{k \in \mathbf{N}}$ of the operator $A$ corresponding to the eigenvalues $\left\{\lambda_{k}^{2}\right\}_{k \in \mathbf{N}}$, that is, $a\left(v_{k}, v\right)=\lambda_{k}^{2}\left(v_{k}\right.$, $v$ ), for all $v \in V$, which is complete in $V$ and orthonormal in $H$. From (11) it follows that the operator $A \in L\left(V ; V^{\prime}\right)$ is invertible and $A^{-1} \in L\left(V^{\prime} ; V\right)$ [47]. Note that $a\left(v_{k}, v\right)=$ $\left\langle A v_{k}, v\right\rangle=\lambda_{k}^{2}\left(v_{k}, v\right)=\left\langle\lambda_{k}^{2} v_{k}, v\right\rangle$ and hence $A v_{k}=\lambda_{k}^{2} v_{k} \in V$, $v_{k} / \lambda_{k}^{2}=A^{-1} v_{k}$ for all $k \in \mathbf{N}$.

Let us define space $V_{1}=\{v \in V ; A v \in H\}$, which is a Hilbert space equipped with the scalar product $(v, w)_{1}=$ ( $A v, A w)$ for all $v, w \in V_{1}$. Space $V_{1}$ is continuously embedded in $V$, since

$$
\begin{aligned}
\|v\|_{V} & =\left\|A^{-1} A v\right\|_{V} \leq\left\|A^{-1}\right\|\|A v\|_{V^{\prime}} \leq c\left\|A^{-1}\right\|\|A v\|_{H} \\
& =c\left\|A^{-1}\right\|\|v\|_{1},
\end{aligned}
$$

where $\|v\|_{1}=\sqrt{(v, v)_{1}}$. Space $V_{1}$ is dense in $V$. Indeed, if $v \in V$, then $A v \in V^{\prime}$. Since $H$ is dense in $V^{\prime}$, there exists sequence $\left(h_{n}\right)_{n \geq 1}, h_{n} \in H, n \in \mathbf{N}$, such that $h_{n} \rightarrow A v$ in $V^{\prime}$, as $n \rightarrow \infty$. Consequently, $A^{-1} h_{n} \rightarrow A^{-1} A v=v$ in $V$ as $n \rightarrow \infty$ and $A^{-1} h_{n} \in V_{1}, n \in \mathbf{N}$.

So, $V_{1} \subset V \subset H \subset V^{\prime} \subset V_{1}^{\prime}$ with continuous and dense embeddings, where $V_{1}^{\prime}$ is the dual space of $V_{1}$. The duality 
relation between $V_{1}^{\prime}$ and $V_{1}$ is denoted by $\langle\cdot, \cdot\rangle_{1}$. Note that for all $v, w \in V_{1}$ we have

$$
\begin{aligned}
|(A v, w)| & \leq\|A v\|_{H}\|w\|_{H}=\|v\|_{1}\|w\|_{H}, \\
\left|\langle A v, w\rangle_{1}\right| & =|(A v, w)|=|a(v, w)|=|a(w, v)| \\
& =|(A w, v)| \leq\|A w\|_{H}\|v\|_{H}=\|v\|_{H}\|w\|_{1} .
\end{aligned}
$$

Hence, $\|A v\|_{H}=\|v\|_{1}$ and $\|A v\|_{V_{1}^{\prime}} \leq c\|v\|_{H}$. Therefore, the restriction of operator $A$ on $V_{1}$ is a linear continuous operator from $V_{1}$ to $H$, and since $V_{1}$ is dense in $H$, there exists unique continuous continuation of $A$ on $H$, which we denote by $A_{1}$ : $H \rightarrow V_{1}^{\prime}, A_{1} v=A v$ for all $v \in V_{1}$. From the definition of the operator $A_{1}$ it follows that

$$
\left\langle A_{1} h, z\right\rangle_{1}=(h, A z) \quad \forall h \in H, z \in V_{1} .
$$

Indeed, since $V_{1}$ is dense in $H$, there exists sequence $\left(z_{n}\right)_{n \geq 1}, z_{n} \in V_{1}, n \in \mathbf{N}$, such that $z_{n} \rightarrow h$ in $H$ as $n \rightarrow \infty$. Consequently, $A z_{n} \rightarrow A_{1} h$ in $V_{1}^{\prime}$ as $n \rightarrow \infty$, and

$$
\begin{aligned}
\left\langle A_{1} h, z\right\rangle_{1} & =\lim _{n \rightarrow \infty}\left(A z_{n}, z\right)=\lim _{n \rightarrow \infty} a\left(z_{n}, z\right) \\
& =\lim _{n \rightarrow \infty} a\left(z, z_{n}\right)=\lim _{n \rightarrow \infty}\left(z_{n}, A z\right)=(h, A z) .
\end{aligned}
$$

In the following lemmas we give some properties of spaces $L^{2}(\Delta ; H), L^{2}(\Delta ; V), L^{2}\left(\Delta ; V^{\prime}\right), L^{2}\left(\Delta ; V_{1}\right)$, and $L^{2}(\Delta$; $\left.V_{1}^{\prime}\right)$, which will be used to prove the main theorem.

Lemma 4. (a) If $g \in L^{2}(\Delta ; H)$, then

$$
\sum_{n=1}^{\infty}\left\|g_{n}\right\|_{L^{2}(\Delta)}^{2} \leq\|g\|_{L^{2}(\Delta ; H)}^{2}, \quad g_{n}=\left(g, v_{n}\right), n \in \mathbf{N}
$$

(b) Suppose that $g_{n} \in L^{2}(\Delta), n \in \mathbf{N}$. The series $\sum_{n=1}^{\infty}\left\|g_{n}\right\|_{L^{2}(\Delta)}^{2}$ converges if and only if the series $\sum_{n=1}^{\infty} g_{n} v_{n}$ converges in the space $L^{2}(\Delta ; H)$.

Proof. (a) Since the system of vector $\left\{v_{k}\right\}_{k \in \mathbf{N}}$ is orthonormal in $H$ we have

$$
\int_{\Delta}\left\|g-\sum_{n=1}^{N} g_{n} v_{n}\right\|_{H}^{2} d \Delta=\|g\|_{L^{2}(\Delta ; H)}^{2}-\sum_{n=1}^{N}\left\|g_{n}\right\|_{L^{2}(\Delta)}^{2} \geq 0
$$

for all $N \in \mathbf{N}$, and tending $N \rightarrow \infty$ we obtain the inequality.

(b) The equivalence of the convergences of the series directly follows from the following equalities:

$$
\begin{aligned}
&\left\|\sum_{n=N}^{M} g_{n} v_{n}\right\|_{L^{2}(\Delta ; H)}^{2}=\int_{\Delta}\left\|\sum_{n=N}^{M} g_{n} v_{n}\right\|_{H}^{2} d \Delta \\
&=\int_{\Delta} \sum_{n=N}^{M} g_{n}^{2} d \Delta=\sum_{n=N}^{M}\left\|g_{n}\right\|_{L^{2}(\Delta)}^{2}, \\
& \forall N, M \in \mathbf{N}, N \leq M .
\end{aligned}
$$

Lemma 5. (a) If $g \in L^{2}(\Delta ; V)$, then

$$
\sum_{n=1}^{\infty} \lambda_{n}^{2}\left\|g_{n}\right\|_{L^{2}(\Delta)}^{2} \leq c_{a}\|g\|_{L^{2}(\Delta ; V)}^{2},
$$

$$
g_{n}=\left(g, v_{n}\right), n \in \mathbf{N} \text {. }
$$

(b) Suppose that $g_{n} \in L^{2}(\Delta), n \in \mathbf{N}$. The series $\sum_{n=1}^{\infty} \lambda_{n}^{2}\left\|g_{n}\right\|_{L^{2}(\Delta)}^{2}$ converges if and only if the series $\sum_{n=1}^{\infty} g_{n} v_{n}$ converges in the space $L^{2}(\Delta ; V)$.

Proof. (a) Since $\left\{v_{k}\right\}_{k \in \mathbf{N}}$ are eigenvectors of the operator $A$ and $\left\{v_{k}\right\}_{k \in \mathbf{N}}$ is orthonormal in $H$ we have

$$
\begin{aligned}
\int_{\Delta} a\left(g-\sum_{n=1}^{N} g_{n} v_{n}, g-\sum_{k=1}^{N} g_{k} v_{k}\right) d \Delta \\
=\int_{\Delta} a(g, g) d \Delta-\int_{\Delta} \sum_{n=1}^{N} \lambda_{n}^{2} g_{n}^{2} d \Delta \geq 0
\end{aligned}
$$

for all $N \in \mathbf{N}$, and tending $N \rightarrow \infty$ from (11) we obtain the required inequality.

(b) The equivalence of the convergences of the series directly follows from the following inequalities for all $N, M \in$ $\mathbf{N}, N \leq M$,

$$
\begin{aligned}
\left\|\sum_{n=N}^{M} g_{n} v_{n}\right\|_{L^{2}(\Delta ; V)}^{2} & \leq \frac{1}{\widehat{c}_{a}} \int_{\Delta} a\left(\sum_{n=N}^{M} g_{n} v_{n}, \sum_{k=N}^{M} g_{k} v_{k}\right) d \Delta \\
& =\frac{1}{\widehat{c}_{a}} \sum_{n=N}^{M} \lambda_{n}^{2}\left\|g_{n}\right\|_{L^{2}(\Delta)}^{2} \\
& \leq \frac{c_{a}}{\widehat{c}_{a}}\left\|\sum_{n=N}^{M} g_{n} v_{n}\right\|_{L^{2}(\Delta ; V)}^{2} .
\end{aligned}
$$

Lemma 6. (a) If $g \in L^{2}\left(\Delta ; V^{\prime}\right)$, then

$$
\begin{aligned}
& \sum_{n=1}^{\infty} \frac{\left\|g_{n}\right\|_{L^{2}(\Delta)}^{2}}{\lambda_{n}^{2}} \leq c_{a}\left\|A^{-1}\right\|^{2}\|g\|_{L^{2}\left(\Delta ; V^{\prime}\right)}^{2}, \\
& \quad g_{n}=\left\langle g, v_{n}\right\rangle, n \in \mathbf{N} .
\end{aligned}
$$

(b) Suppose that $g_{n} \in L^{2}(\Delta), n \in \mathbf{N}$. The series $\sum_{n=1}^{\infty}\left\|g_{n}\right\|_{L^{2}(\Delta)}^{2} / \lambda_{n}^{2}$ converges if and only if the series $\sum_{n=1}^{\infty} g_{n} v_{n}$ converges in the space $L^{2}\left(\Delta ; V^{\prime}\right)$.

Proof. (a) Let us define $\hat{g}=A^{-1} g \in L^{2}(\Delta ; V)$. Applying part (a) of Lemma 5 we have $\sum_{n=1}^{\infty} \lambda_{n}^{2}\left\|\widehat{g}_{n}\right\|_{L^{2}(\Delta)}^{2} \leq c_{a}\|\hat{g}\|_{L^{2}(\Delta ; V)}^{2}$, where

$$
\begin{aligned}
\widehat{g}_{n} & =\left(\hat{g}, v_{n}\right)=\left(v_{n}, \widehat{g}\right)=\frac{a\left(v_{n}, \widehat{g}\right)}{\lambda_{n}^{2}}=\frac{a\left(\widehat{g}, v_{n}\right)}{\lambda_{n}^{2}} \\
& =\frac{\left\langle A \hat{g}, v_{n}\right\rangle}{\lambda_{n}^{2}}=\frac{\left\langle g, v_{n}\right\rangle}{\lambda_{n}^{2}}=\frac{g_{n}}{\lambda_{n}^{2}}, \quad \forall n \in \mathbf{N} .
\end{aligned}
$$


Consequently,

$$
\begin{aligned}
\sum_{n=1}^{\infty} \lambda_{n}^{2}\left\|\widehat{g}_{n}\right\|_{L^{2}(\Delta)}^{2} & =\sum_{n=1}^{\infty} \frac{\left\|g_{n}\right\|_{L^{2}(\Delta)}^{2}}{\lambda_{n}^{2}} \leq c_{a}\left\|A^{-1} g\right\|_{L^{2}(\Delta ; V)}^{2} \\
& \leq c_{a}\left\|A^{-1}\right\|^{2}\|g\|_{L^{2}\left(\Delta ; V^{\prime}\right)}^{2} .
\end{aligned}
$$

(b) The equivalence of the convergences of the series directly follows from the following inequalities

$$
\begin{aligned}
& \left\|\sum_{n=N}^{M} g_{n} v_{n}\right\|_{L^{2}\left(\Delta ; V^{\prime}\right)}^{2}=\left\|A A^{-1} \sum_{n=N}^{M} g_{n} v_{n}\right\|_{L^{2}\left(\Delta ; V^{\prime}\right)}^{2} \\
& \leq\|A\|^{2}\left\|\sum_{n=N}^{M} g_{n} A^{-1} v_{n}\right\|_{L^{2}(\Delta ; V)}^{2} \\
& \leq \frac{\|A\|^{2}}{\widehat{c}_{a}} \int_{\Delta} a\left(\sum_{n=N}^{M} g_{n} \frac{v_{n}}{\lambda_{n}^{2}}, \sum_{k=N}^{M} g_{k} \frac{v_{k}}{\lambda_{k}^{2}}\right) d \Delta \\
& =\frac{\|A\|^{2}}{\widehat{c}_{a}} \int_{\Delta} \sum_{n=N}^{M} \frac{g_{n}^{2}}{\lambda_{n}^{2}} d \Delta \\
& \leq \frac{\|A\|^{2}}{\widehat{c}_{a}} c_{a} \int_{\Delta}\left\|\sum_{n=N}^{M} g_{n} \frac{v_{n}}{\lambda_{n}^{2}}\right\|_{V}^{2} d \Delta \\
& \quad=\frac{\|A\|^{2}}{\widehat{c}_{a}} c_{a} \int_{\Delta}\left\|\sum_{n=N}^{M} g_{n} A^{-1} v_{n}\right\|_{V}^{2} d \Delta \\
& \leq \frac{\|A\|^{2}}{\widehat{c}_{a}} c_{a}\left\|A^{-1} \sum_{n=N}^{M} g_{n} v_{n}\right\|_{L^{2}(\Delta ; V)} \widehat{c}_{a} A^{-1}\left\|^{2}\right\| \sum_{n=N}^{M} g_{n} v_{n} \|_{L^{2}\left(\Delta ; V^{\prime}\right)}^{2} \quad \forall N, M \in \mathbf{N}, N \leq M . \\
& \leq
\end{aligned}
$$

Lemma 7. (a) If $g \in L^{2}\left(\Delta ; V_{1}\right)$, then

$$
\sum_{n=1}^{\infty} \lambda_{n}^{4}\left\|g_{n}\right\|_{L^{2}(\Delta)}^{2} \leq\|g\|_{L^{2}\left(\Delta ; V_{1}\right)}^{2}, \quad g_{n}=\left(g, v_{n}\right), n \in \mathbf{N}
$$

(b) Suppose that $g_{n} \in L^{2}(\Delta), n \in \mathbf{N}$. The series $\sum_{n=1}^{\infty} \lambda_{n}^{4}\left\|g_{n}\right\|_{L^{2}(\Delta)}^{2}$ converges if and only if the series $\sum_{n=1}^{\infty} g_{n} v_{n}$ converges in the space $L^{2}\left(\Delta ; V_{1}\right)$.

Proof. (a) Since $\left\{v_{k}\right\}_{k \in \mathbf{N}}$ are eigenvectors of operator $A$ and $\left\{v_{k}\right\}_{k \in \mathbf{N}}$ is orthonormal in $H$ we have $\left(A g, v_{k}\right)=a\left(g, v_{k}\right)=$ $a\left(v_{k}, g\right)=\lambda_{k}^{2} g_{k}$ for all $k \in \mathbf{N}$, and hence

$$
\begin{aligned}
& \int_{\Delta}\left(A g-\sum_{n=1}^{N} g_{n} A v_{n}, A g-\sum_{k=1}^{N} g_{k} A v_{k}\right) d \Delta \\
& =\int_{\Delta}\left(A g-\sum_{n=1}^{N} g_{n} \lambda_{n}^{2} v_{n}, A g-\sum_{k=1}^{N} g_{k} \lambda_{k}^{2} v_{k}\right) d \Delta
\end{aligned}
$$

$$
=\int_{\Delta}(A g, A g) d \Delta-\int_{\Delta} \sum_{n=1}^{N} \lambda_{n}^{4} g_{n}^{2} d \Delta \geq 0
$$

for all $N \in \mathbf{N}$, and tending $N \rightarrow \infty$ from the definition of the norm $\|\cdot\|_{1}$ we obtain the required inequality.

(b) The equivalence of the convergences of the series directly follows from the following inequalities:

$$
\begin{gathered}
\left\|\sum_{n=N}^{M} g_{n} v_{n}\right\|_{L^{2}\left(\Delta ; V_{1}\right)}^{2}=\int_{\Delta}\left(\sum_{n=N}^{M} g_{n} A v_{n}, \sum_{k=N}^{M} g_{k} A v_{k}\right) d \Delta \\
\quad=\int_{\Delta}\left(\sum_{n=N}^{M} g_{n} \lambda_{n}^{2} v_{n}, \sum_{k=N}^{M} g_{k} \lambda_{k}^{2} v_{k}\right) d \Delta \\
=\sum_{n=N}^{M} \lambda_{n}^{4}\left\|g_{n}\right\|_{L^{2}(\Delta)}^{2}, \quad \forall N, M \in \mathbf{N}, \quad N \leq M .
\end{gathered}
$$

Lemma 8. (a) If $g \in L^{2}\left(\Delta ; V_{1}^{\prime}\right)$, then

$$
\begin{aligned}
& \sum_{n=1}^{\infty} \frac{\left\|g_{n}\right\|_{L^{2}(\Delta)}^{2}}{\lambda_{n}^{4}} \leq\left\|\left(A_{1} A\right)^{-1}\right\|^{2}\|g\|_{L^{2}\left(\Delta ; V_{1}^{\prime}\right)}^{2}, \\
& \qquad g_{n}=\left\langle g, v_{n}\right\rangle_{1}, n \in \mathbf{N} .
\end{aligned}
$$

(b) Suppose that $g_{n} \in L^{2}(\Delta), n \in \mathbf{N}$. The series $\sum_{n=1}^{\infty}\left\|g_{n}\right\|_{L^{2}(\Delta)}^{2} / \lambda_{n}^{4}$ converges if and only if the series $\sum_{n=1}^{\infty} g_{n} v_{n}$ converges in the space $L^{2}\left(\Delta ; V_{1}^{\prime}\right)$.

Proof. (a) Note that the operator $B=A_{1} A: V_{1} \rightarrow V_{1}^{\prime}$ is linear and continuous, and applying (14) we obtain that $B$ satisfies the following coerciveness condition:

$$
\langle B z, z\rangle_{1}=\left\langle A_{1} A z, z\right\rangle_{1}=(A z, A z)=\|z\|_{1}^{2} \quad \forall z \in V_{1} .
$$

Consequently, the operator $B \in L\left(V_{1} ; V_{1}^{\prime}\right)$ is invertible and $B^{-1} \in L\left(V_{1}^{\prime} ; V_{1}\right)$ [47].

Let us define $\hat{g}=B^{-1} g \in L^{2}\left(\Delta ; V_{1}\right)$. Applying part (a) of Lemma 7 we have $\sum_{n=1}^{\infty} \lambda_{n}^{4}\left\|\widehat{g}_{n}\right\|_{L^{2}(\Delta)}^{2} \leq\|\hat{g}\|_{L^{2}\left(\Delta ; V_{1}\right)}^{2}$ and from equality (14) we obtain

$$
\begin{aligned}
\widehat{g}_{n} & =\left(\widehat{g}, v_{n}\right)=\left(v_{n}, \widehat{g}\right)=\frac{a\left(v_{n}, \widehat{g}\right)}{\lambda_{n}^{2}}=\frac{a\left(\hat{g}, v_{n}\right)}{\lambda_{n}^{2}} \\
& =\frac{\left(A \widehat{g}, v_{n}\right)}{\lambda_{n}^{2}}=\frac{\left(A \widehat{g}, A v_{n}\right)}{\lambda_{n}^{4}}=\frac{\left\langle A_{1} A \widehat{g}, v_{n}\right\rangle_{1}}{\lambda_{n}^{4}} \\
& =\frac{\left\langle g, v_{n}\right\rangle_{1}}{\lambda_{n}^{4}}=\frac{g_{n}}{\lambda_{n}^{4}}
\end{aligned}
$$

for all $n \in \mathbf{N}$. Consequently,

$$
\begin{aligned}
\sum_{n=1}^{\infty} \lambda_{n}^{4}\left\|\widehat{g}_{n}\right\|_{L^{2}(\Delta)}^{2} & =\sum_{n=1}^{\infty} \frac{\left\|g_{n}\right\|_{L^{2}(\Delta)}^{2}}{\lambda_{n}^{4}} \leq\left\|\left(A_{1} A\right)^{-1} g\right\|_{L^{2}\left(\Delta ; V_{1}\right)}^{2} \\
& \leq\left\|\left(A_{1} A\right)^{-1}\right\|^{2}\|g\|_{L^{2}\left(\Delta ; V_{1}^{\prime}\right)}^{2} .
\end{aligned}
$$


(b) Since $B v_{n}=A_{1} A v_{n}=\lambda_{n}^{2} A_{1} v_{n}=\lambda_{n}^{2} A v_{n}=\lambda_{n}^{4} v_{n}$, we have $v_{n} / \lambda_{n}^{4}=B^{-1} v_{n}$ and the equivalence of the convergences of the series directly follows from the following inequalities:

$$
\begin{aligned}
\left\|\sum_{n=N}^{M} g_{n} v_{n}\right\|_{L^{2}\left(\Delta ; V_{1}^{\prime}\right)}^{2} & =\left\|B B^{-1} \sum_{n=N}^{M} g_{n} v_{n}\right\|_{L^{2}\left(\Delta ; V_{1}^{\prime}\right)}^{2} \\
& \leq\|B\|^{2}\left\|\sum_{n=N}^{M} g_{n} B^{-1} v_{n}\right\|_{L^{2}\left(\Delta ; V_{1}\right)}^{2} \\
& =\|B\|^{2}\left\|\sum_{n=N}^{M} g_{n} \frac{v_{n}}{\lambda_{n}^{4}}\right\|_{L^{2}\left(\Delta ; V_{1}\right)}^{2} \\
& =\|B\|^{2}\left\|A \sum_{n=N}^{M} g_{n} \frac{v_{n}}{\lambda_{n}^{4}}\right\|_{L^{2}(\Delta ; H)}^{2} \\
& =\|B\|^{2}\left\|\sum_{n=N}^{M} g_{n} \frac{v_{n}}{\lambda_{n}^{2}}\right\|_{L^{2}(\Delta ; H)}^{2} \\
& =\|B\|^{2} \int_{\Delta} \sum_{n=N}^{M} \frac{g_{n}^{2}}{\lambda_{n}^{4}} d \Delta \\
& \leq\|B\|^{2}\left\|B^{-1}\right\|^{2}\left\|\sum_{n=N}^{M} g_{n} v_{n}\right\|_{L^{2}\left(\Delta ; V_{1}^{\prime}\right)}^{2}
\end{aligned}
$$

for all $N, M \in \mathbf{N}, N \leq M$.

\section{Main Result}

In the present paper we investigate nonlocal problem (1), (2) applying the following variational formulation: find a vectorfunction $u \in W, d\left(\operatorname{tr}_{t_{2}=0} u\right) / d t_{1} \in L^{2}\left(0, T_{1} ; V_{1}^{\prime}\right), d\left(\operatorname{tr}_{t_{1}=0} u\right) /$ $d t_{2} \in L^{2}\left(0, T_{2} ; V_{1}^{\prime}\right)$, which satisfies equation

$$
\frac{\partial}{\partial t_{1}}(u, v)+\frac{\partial}{\partial t_{2}}(u, v)+a(u, v)=\langle f, v\rangle, \quad \forall v \in V,
$$

in the sense of distributions on $\omega$, and initial conditions

$$
\begin{aligned}
& \left(\operatorname{tr}_{t_{1}=0} u\right)\left(t_{2}\right)=\varphi\left(t_{2}\right), \quad 0 \leq t_{2} \leq T_{2}, \\
& \left(\operatorname{tr}_{t_{2}=0} u\right)\left(t_{1}\right)=\alpha\left(\operatorname{tr}_{t_{2}=\xi} u\right)\left(t_{1}\right)+\psi\left(t_{1}\right),
\end{aligned}
$$

$$
0 \leq t_{1} \leq T_{1},
$$

in the spaces $L^{2}\left(0, T_{2} ; H\right)$ and $L^{2}\left(0, T_{1} ; H\right)$, respectively. Applying Theorem 3 and by taking account of the density of linear combinations of products $\phi v, \phi \in D(\omega), v \in V$, in $L^{2}(\omega ; V)$ we obtain that (35) is equivalent to (1), which is considered as equation in the space $L^{2}\left(\omega ; V^{\prime}\right)$.

Let us prove the following lemmas, which will be used to obtain the existence of solution of problem (34), (35). We denote by $\omega_{1}=\left\{\left(t_{1}, t_{2}\right) \in \omega ; t_{2}>t_{1}\right\}$ and $\omega_{2}=\left\{\left(t_{1}, t_{2}\right) \in\right.$ $\left.\omega ; t_{1}>t_{2}\right\}$ the parts of the rectangle $\omega$ above and below the bisector $\left\{\left(t_{1}, t_{2}\right) ; t_{1}=t_{2}\right\}$, respectively; $\bar{\omega}_{1}$ and $\bar{\omega}_{2}$ are closures of $\omega_{1}$ and $\omega_{2}$.

Lemma 9. If $g \in L^{2}\left(0, T_{2}\right)$ and $\tilde{g} \in L^{2}\left(0, T_{1}\right)$, then the following estimates are valid

$$
\begin{aligned}
& \int_{\omega_{1}} e^{-2 \lambda_{n}^{2} t_{1}} g^{2}\left(t_{2}-t_{1}\right) d t_{1} d t_{2} \leq \frac{1}{2 \lambda_{n}^{2}} \int_{0}^{T_{2}} g^{2}(s) d s, \\
& \int_{\omega_{2}} e^{-2 \lambda_{n}^{2} t_{2}} g^{2}\left(t_{1}-t_{2}\right) d t_{1} d t_{2} \leq \frac{1}{2 \lambda_{n}^{2}} \int_{0}^{T_{1}} g^{2}(s) d s .
\end{aligned}
$$

Proof. Taking into account the definition of the sets $\omega_{1}$ and $\omega_{2}$ we obtain

$$
\begin{gathered}
\int_{\omega_{1}} e^{-2 \lambda_{n}^{2} t_{1}} g^{2}\left(t_{2}-t_{1}\right) d t_{1} d t_{2} \\
\leq \int_{0}^{T_{2}} \int_{0}^{t_{2}} e^{-2 \lambda_{n}^{2}\left(t_{2}-s\right)} g^{2}(s) d s d t_{2} \\
=\int_{0}^{T_{2}} \int_{s}^{T_{2}} e^{-2 \lambda_{n}^{2}\left(t_{2}-s\right)} g^{2}(s) d s d t_{2} \\
=\int_{0}^{T_{2}} \frac{1-e^{-2 \lambda_{n}^{2}\left(T_{2}-s\right)}}{2 \lambda_{n}^{2}} g^{2}(s) d s,
\end{gathered}
$$

which implies the first estimate. Likewise we obtain the second estimate.

Lemma 10. If $g \in H^{1}(\omega)$, then the following estimates are valid

$$
\begin{aligned}
& \int_{\omega_{1}}\left(\int_{0}^{t_{1}} e^{-\lambda_{n}^{2}\left(t_{1}-\tau\right)} g\left(\tau, \tau+t_{2}-t_{1}\right) d \tau\right)^{2} d t_{1} d t_{2} \\
& \quad \leq \frac{c}{\lambda_{n}^{4}}\|g\|_{H^{1}(\omega)}^{2}, \\
& \int_{\omega_{2}}\left(\int_{0}^{t_{2}} e^{-\lambda_{n}^{2}\left(t_{2}-\tau\right)} g\left(\tau+t_{1}-t_{2}, \tau\right) d \tau\right)^{2} d t_{1} d t_{2} \\
& \quad \leq \frac{c}{\lambda_{n}^{4}}\|g\|_{H^{1}(\omega)}^{2} .
\end{aligned}
$$

Proof. Assuming that $g \in C^{1}(\bar{\omega}), \bar{\omega}=\left[0, T_{1}\right] \times\left[0, T_{2}\right]$, we have

$$
\begin{aligned}
& \int_{\omega_{1}}\left(\int_{0}^{t_{1}} e^{-\lambda_{n}^{2}\left(t_{1}-\tau\right)} g\left(\tau, \tau+t_{2}-t_{1}\right) d \tau\right)^{2} d t_{1} d t_{2} \\
& \quad=\int_{\omega_{1}}\left(\frac{g\left(t_{1}, t_{2}\right)}{\lambda_{n}^{2}}-\frac{e^{-\lambda_{n}^{2} t_{1}} g\left(0, t_{2}-t_{1}\right)}{\lambda_{n}^{2}}-\int_{0}^{t_{1}} \frac{e^{-\lambda_{n}^{2}\left(t_{1}-\tau\right)}}{\lambda_{n}^{2}}\left(\frac{\partial g}{\partial \tau_{1}}\left(\tau, \tau+t_{2}-t_{1}\right)+\frac{\partial g}{\partial \tau_{2}}\left(\tau, \tau+t_{2}-t_{1}\right)\right) d \tau\right)^{2} d t_{1} d t_{2}
\end{aligned}
$$




$$
\begin{aligned}
& \leq \frac{4}{\lambda_{n}^{4}} \int_{\omega_{1}} g^{2}\left(t_{1}, t_{2}\right) d t_{1} d t_{2}+\frac{4}{\lambda_{n}^{4}} \int_{0}^{T_{2}} \int_{0}^{t_{2}} g^{2}(0, s) d s d t_{2} \\
& +\frac{4 T_{1} T_{2}}{\lambda_{n}^{4}}\left[\int_{\omega_{1}}\left(\frac{\partial g}{\partial \tau_{1}}\left(t_{1}, t_{2}\right)\right)^{2} d t_{1} d t_{2}+\int_{\omega_{1}}\left(\frac{\partial g}{\partial \tau_{2}}\left(t_{1}, t_{2}\right)\right)^{2} d t_{1} d t_{2}\right] .
\end{aligned}
$$

From continuity of the trace operator $H^{1}(\omega) \rightarrow L^{2}(\partial \omega)$ we obtain

$$
\begin{aligned}
& \int_{0}^{T_{2}} \int_{0}^{t_{2}} g^{2}(0, s) d s d t_{2} \leq T_{2} \int_{0}^{T_{2}} g^{2}(0, s) d s \\
& \quad \leq T_{2} c\|g\|_{H^{1}(\omega)}^{2} .
\end{aligned}
$$

The latter inequality implies the first estimate of the lemma for functions from $C^{1}(\bar{\omega})$ and since $C^{1}(\bar{\omega})$ is dense in $H^{1}(\omega)$, by passing to the limit we obtain the estimate for functions from $H^{1}(\omega)$. Likewise we obtain the second estimate.

Lemma 11. If $g \in H^{1}(\omega)$, then the following estimates are valid

$$
\begin{aligned}
& \int_{0}^{\xi_{T_{1}}}\left(\int_{0}^{t_{1}} e^{-\lambda_{n}^{2}\left(t_{1}-\tau\right)} g\left(\tau, \tau+\xi-t_{1}\right) d \tau\right)^{2} d t_{1} \\
& \quad \leq \frac{c}{\lambda_{n}^{4}}\|g\|_{H^{1}(\omega)}^{2}, \\
& \int_{\xi_{T_{1}}}^{T_{1}}\left(\int_{0}^{\xi} e^{-\lambda_{n}^{2}(\xi-\tau)} g\left(\tau+t_{1}-\xi, \tau\right) d \tau\right)^{2} d t_{1} \\
& \quad \leq \frac{c}{\lambda_{n}^{4}}\|g\|_{H^{1}(\omega)}^{2},
\end{aligned}
$$

where $\xi_{T_{1}}=\min \left\{\xi, T_{1}\right\}$.

Proof. Assuming that $g \in C^{1}(\bar{\omega})$ we get

$$
\begin{aligned}
& \int_{0}^{\xi_{T_{1}}}\left(\int_{0}^{t_{1}} e^{-\lambda_{n}^{2}\left(t_{1}-\tau\right)} g\left(\tau, \tau+\xi-t_{1}\right) d \tau\right)^{2} d t_{1} \\
& \quad=\int_{0}^{\xi_{T_{1}}}\left(\frac{g\left(t_{1}, \xi\right)}{\lambda_{n}^{2}}-\frac{e^{-\lambda_{n}^{2} t_{1}} g\left(0, \xi-t_{1}\right)}{\lambda_{n}^{2}}-\int_{0}^{t_{1}} \frac{e^{-\lambda_{n}^{2}\left(t_{1}-\tau\right)}}{\lambda_{n}^{2}}\left(\frac{\partial g}{\partial \tau_{1}}\left(\tau, \tau+\xi-t_{1}\right)+\frac{\partial g}{\partial \tau_{2}}\left(\tau, \tau+\xi-t_{1}\right)\right) d \tau\right) d t_{1} \leq \frac{4}{\lambda_{n}^{4}} \\
& \quad \cdot \int_{0}^{\xi_{T_{1}}} g^{2}\left(t_{1}, \xi\right) d t_{1}+\frac{4}{\lambda_{n}^{4}} \int_{0}^{\xi} g^{2}(0, s) d s+\frac{4 T_{1}}{\lambda_{n}^{4}} \int_{\omega_{1}}\left[\left(\frac{\partial g}{\partial \tau_{1}}\right)^{2}+\left(\frac{\partial g}{\partial \tau_{2}}\right)^{2}\right] d t_{1} d t_{2} .
\end{aligned}
$$

The continuity of the trace operator $H^{1}\left(\omega_{\xi}\right) \rightarrow L^{2}\left(\partial \omega_{\xi}\right), \omega_{\xi}=$ $\left(0, T_{1}\right) \times(0, \xi)$, implies that

$$
\begin{aligned}
& \max \left\{\int_{0}^{\xi_{T_{1}}} g^{2}\left(t_{1}, \xi\right) d t_{1}, \int_{0}^{\xi} g^{2}(0, s) d s\right\} \\
& \leq c\|g\|_{H^{1}\left(\omega_{\xi}\right)}^{2} \leq c\|g\|_{H^{1}(\omega)}^{2} .
\end{aligned}
$$

From the latter inequality we obtain the first estimate of the lemma for functions from $C^{1}(\bar{\omega})$ and since $C^{1}(\bar{\omega})$ is dense in $H^{1}(\omega)$, by passing to the limit we obtain the estimate for functions from $H^{1}(\omega)$. Likewise we obtain the second estimate.

The following existence and uniqueness theorem is valid for nonlocal problem (34), (35).

Theorem 12. If $\varphi \in L^{2}\left(0, T_{2} ; H\right), \varphi^{\prime} \in L^{2}\left(0, T_{2} ; V_{1}^{\prime}\right)$, $\psi \in L^{2}\left(0, T_{1} ; H\right), \psi^{\prime} \in L^{2}\left(0, T_{1} ; V_{1}^{\prime}\right), \varphi$ and $\psi$ satisfy compatibility condition $\varphi(0)=\alpha \varphi(\xi)+\psi(0)$ and $f, \partial f / \partial t_{1}, \partial f / \partial t_{2} \in L^{2}\left(\omega ; V^{\prime}\right)$, then problem (34), (35) has a unique solution and the following estimate is valid:

$$
\begin{aligned}
\|u\|_{W} & \leq c\left(\|\varphi\|_{L^{2}\left(0, T_{2} ; H\right)}+\left\|\varphi^{\prime}\right\|_{L^{2}\left(0, T_{2} ; V_{1}^{\prime}\right)}+\|\psi\|_{L^{2}\left(0, T_{1} ; H\right)}\right. \\
+ & \left\|\psi^{\prime}\right\|_{L^{2}\left(0, T_{1} ; V_{1}^{\prime}\right)}+\|f\|_{L^{2}\left(\omega ; V^{\prime}\right)}+\left\|\frac{\partial f}{\partial t_{1}}\right\|_{L^{2}\left(\omega ; V^{\prime}\right)} \\
+ & \left.\left\|\frac{\partial f}{\partial t_{2}}\right\|_{L^{2}\left(\omega ; V^{\prime}\right)}\right) .
\end{aligned}
$$

Proof. Applying embedding theorem [46] we have that $\varphi \in$ $C\left(\left[0, T_{2}\right] ; V^{\prime}\right), \psi \in C\left(\left[0, T_{1}\right] ; V^{\prime}\right)$ and the compatibility condition is the equality in $V^{\prime}$. First, we prove the existence of a solution. Let us consider the sequence of approximate solutions $w_{N}\left(t_{1}, t_{2}\right)=\sum_{n=1}^{N} u_{n}\left(t_{1}, t_{2}\right) v_{n}$, where $u_{n}$ is a solution of the following nonlocal problem for the first-order partial differential equation:

$$
\frac{\partial u_{n}}{\partial t_{1}}+\frac{\partial u_{n}}{\partial t_{2}}+\lambda_{n}^{2} u_{n}=f_{n}, \quad\left(t_{1}, t_{2}\right) \in \omega,
$$




$$
\begin{aligned}
& u_{n}\left(0, t_{2}\right)=\varphi_{n}\left(t_{2}\right), \quad 0 \leq t_{2} \leq T_{2}, \\
& u_{n}\left(t_{1}, 0\right)=\alpha u_{n}\left(t_{1}, \xi\right)+\psi_{n}\left(t_{1}\right), \\
& \quad 0 \leq t_{1} \leq T_{1},
\end{aligned}
$$

where $\varphi_{n}=\left(\varphi, v_{n}\right), \psi_{n}=\left(\psi, v_{n}\right), f_{n}=\left\langle f, v_{n}\right\rangle, n \in \mathbf{N}$. Note that $\varphi_{n} \in H^{1}\left(0, T_{2}\right), \psi_{n} \in H^{1}\left(0, T_{1}\right), f_{n} \in H^{1}(\omega)$ and hence $\varphi_{n} \in C\left[0, T_{2}\right], \psi_{n} \in C\left[0, T_{1}\right]$.

Let us denote by $g_{1 n}$ and $g_{2 n}$ the following functions:

$$
\begin{array}{r}
g_{1 n}\left(t_{1}, t_{2}\right)=\int_{0}^{t_{1}} e^{-\lambda_{n}^{2}\left(t_{1}-\tau\right)} f_{n}\left(\tau, \tau+t_{2}-t_{1}\right) d \tau, \\
\left(t_{1}, t_{2}\right) \in \bar{\omega}_{1}, \\
g_{2 n}\left(t_{1}, t_{2}\right)=\int_{0}^{t_{2}} e^{-\lambda_{n}^{2}\left(t_{2}-\tau\right)} f_{n}\left(\tau+t_{1}-t_{2}, \tau\right) d \tau, \\
\left(t_{1}, t_{2}\right) \in \bar{\omega}_{2} .
\end{array}
$$

Functions $g_{1 n}$ and $g_{2 n}$ are defined for all $\left(t_{1}, t_{2}\right) \in \bar{\omega}_{1}$ and $\left(t_{1}, t_{2}\right) \in \bar{\omega}_{2}$, respectively, because the trace of $f_{n}$ on each line

$$
l_{t_{1} t_{2}}= \begin{cases}\left(\tau, \tau+t_{2}-t_{1}\right) ; & 0<\tau<t_{1} \text { for } t_{2} \geq t_{1} \\ \left(\tau+t_{1}-t_{2}, \tau\right) ; & 0<\tau<t_{2} \text { for } t_{1} \geq t_{2}\end{cases}
$$

which is parallel to the bisector $\left\{\left(t_{1}, t_{2}\right) ; t_{1}=t_{2}\right\}$, which belongs to $L^{2}\left(l_{t_{1} t_{2}}\right)$.

Note that $g_{1 n} \in L^{2}\left(\omega_{1}\right)$ and $g_{2 n} \in L^{2}\left(\omega_{2}\right)$. Their generalized derivative is given by

$$
\begin{aligned}
\frac{\partial g_{1 n}}{\partial t_{1}}= & f_{n}\left(t_{1}, t_{2}\right) \\
& -\lambda_{n}^{2} \int_{0}^{t_{1}} e^{-\lambda_{n}^{2}\left(t_{1}-\tau\right)} f_{n}\left(\tau, \tau+t_{2}-t_{1}\right) d \tau \\
& -\int_{0}^{t_{1}} e^{-\lambda_{n}^{2}\left(t_{1}-\tau\right)} \frac{\partial f_{n}}{\partial \tau_{2}}\left(\tau, \tau+t_{2}-t_{1}\right) d \tau, \\
\frac{\partial g_{1 n}}{\partial t_{2}}= & \int_{0}^{t_{1}} e^{-\lambda_{n}^{2}\left(t_{1}-\tau\right)} \frac{\partial f_{n}}{\partial \tau_{2}}\left(\tau, \tau+t_{2}-t_{1}\right) d \tau
\end{aligned}
$$

for almost all $\left(t_{1}, t_{2}\right) \in \omega_{1}$,

$$
\begin{aligned}
\frac{\partial g_{2 n}}{\partial t_{1}}= & \int_{0}^{t_{2}} e^{-\lambda_{n}^{2}\left(t_{2}-\tau\right)} \frac{\partial f_{n}}{\partial \tau_{1}}\left(\tau+t_{1}-t_{2}, \tau\right) d \tau \\
\frac{\partial g_{2 n}}{\partial t_{2}}= & f_{n}\left(t_{1}, t_{2}\right) \\
& -\lambda_{n}^{2} \int_{0}^{t_{2}} e^{-\lambda_{n}^{2}\left(t_{2}-\tau\right)} f_{n}\left(\tau+t_{1}-t_{2}, \tau\right) d \tau \\
& +\int_{0}^{t_{2}} e^{-\lambda_{n}^{2}\left(t_{2}-\tau\right)} \frac{\partial f_{n}}{\partial \tau_{1}}\left(\tau+t_{1}-t_{2}, \tau\right) d \tau
\end{aligned}
$$

for almost all $\left(t_{1}, t_{2}\right) \in \omega_{2}$. Applying Lemma 10 we obtain

$$
\begin{aligned}
& \left\|\frac{\partial g_{1 n}}{\partial t_{1}}\right\|_{L^{2}\left(\omega_{1}\right)}^{2} \leq 3\left\|f_{n}\right\|_{L^{2}\left(\omega_{1}\right)}^{2}+3 c\left\|f_{n}\right\|_{H^{1}(\omega)}^{2} \\
& \quad+T_{1} \int_{0}^{T_{2}} \int_{0}^{\min \left\{t_{2}, T_{1}\right\}} \int_{0}^{t_{1}}\left(\frac{\partial f_{n}}{\partial \tau_{2}}\right)^{2}\left(\tau, \tau+t_{2}-t_{1}\right) d \tau d t_{1} d t_{2} \\
& \quad \leq 3(c+1)\left\|f_{n}\right\|_{H^{1}(\omega)}^{2}+T_{1} T_{2} \int_{\omega_{1}}\left(\frac{\partial f_{n}}{\partial \tau_{2}}\right)^{2} d t_{1} d t_{2} \\
& \quad \leq c\left\|f_{n}\right\|_{H^{1}(\omega)}^{2}, \\
& \left\|\frac{\partial g_{1 n}}{\partial t_{2}}\right\|_{L^{2}\left(\omega_{1}\right)}^{2} \\
& \quad \leq T_{1} \int_{0}^{T_{2}} \int_{0}^{\min \left\{t_{2}, T_{1}\right\}} \int_{0}^{t_{1}}\left(\frac{\partial f_{n}}{\partial \tau_{2}}\right)^{2}\left(\tau, \tau+t_{2}-t_{1}\right) d \tau d t_{1} d t_{2} \\
& \quad \leq T_{1} T_{2} \int_{\omega_{1}}\left(\frac{\partial f_{n}}{\partial \tau_{2}}\right)^{2} d t_{1} d t_{2} \leq T_{1} T_{2}\left\|f_{n}\right\|_{H^{1}(\omega)}^{2},
\end{aligned}
$$

$$
\begin{aligned}
& \left\|\frac{\partial g_{2 n}}{\partial t_{1}}\right\|_{L^{2}\left(\omega_{2}\right)}^{2} \\
& \quad \leq T_{2} \int_{0}^{T_{1}} \int_{0}^{\min \left\{t_{1}, T_{2}\right\}} \int_{0}^{t_{2}}\left(\frac{\partial f_{n}}{\partial \tau_{1}}\right)^{2}\left(\tau+t_{1}-t_{2}, \tau\right) d \tau d t_{2} d t_{1} \\
& \quad \leq T_{1} T_{2} \int_{\omega_{2}}\left(\frac{\partial f_{n}}{\partial \tau_{1}}\right)^{2} d t_{1} d t_{2} \leq T_{1} T_{2}\left\|f_{n}\right\|_{H^{1}(\omega)}^{2}, \\
& \left\|\frac{\partial g_{2 n}}{\partial t_{2}}\right\|_{L^{2}\left(\omega_{2}\right)}^{2} \leq 3\left\|f_{n}\right\|_{L^{2}\left(\omega_{2}\right)}^{2}+3 c\left\|f_{n}\right\|_{H^{1}(\omega)}^{2} \\
& \quad+T_{2} \int_{0}^{T_{1}} \int_{0}^{\min \left\{t_{1}, T_{2}\right\}} \int_{0}^{t_{2}}\left(\frac{\partial f_{n}}{\partial \tau_{1}}\right)^{2}\left(\tau+t_{1}-t_{2}, \tau\right) d \tau d t_{2} d t_{1} \\
& \quad \leq 3(c+1)\left\|f_{n}\right\|_{H^{1}(\omega)}^{2}+T_{2} T_{1} \int_{\omega_{2}}\left(\frac{\partial f_{n}}{\partial \tau_{1}}\right)^{2} d t_{1} d t_{2} \\
& \quad \leq c\left\|f_{n}\right\|_{H^{1}(\omega)}^{2} \cdot
\end{aligned}
$$

Therefore, $g_{1 n} \in H^{1}\left(\omega_{1}\right)$ and $g_{2 n} \in H^{1}\left(\omega_{2}\right)$.

Applying Lemma 11 and continuity of the trace operator $H^{1}\left(\omega_{\xi}\right) \rightarrow L^{2}\left(\partial \omega_{\xi}\right)$ we obtain

$$
\begin{aligned}
& \left\|g_{1 n}\left(t_{1}, \xi\right)\right\|_{L^{2}\left(0, \xi_{T_{1}}\right)}^{2} \leq \frac{c}{\lambda_{n}^{4}}\left\|f_{n}\right\|_{H^{1}(\omega)}^{2}, \\
& \left\|g_{2 n}\left(t_{1}, \xi\right)\right\|_{L^{2}\left(\xi, T_{1}\right)}^{2} \leq \frac{c}{\lambda_{n}^{4}}\left\|f_{n}\right\|_{H^{1}(\omega)}^{2} \quad \text { for } T_{1}>\xi, \\
& \left\|\frac{d g_{1 n}\left(t_{1}, \xi\right)}{d t_{1}}\right\|_{L^{2}\left(0, \xi_{T_{1}}\right)}^{2} \leq 3\left\|f_{n}\left(t_{1}, \xi\right)\right\|_{L^{2}\left(0, \xi_{T_{1}}\right)}^{2} \\
& \quad+3 \lambda_{n}^{4} \int_{0}^{\xi_{T_{1}}}\left(\int_{0}^{t_{1}} e^{-\lambda_{n}^{2}\left(t_{1}-\tau\right)} f_{n}\left(\tau, \tau+\xi-t_{1}\right) d \tau\right)^{2} d t_{1} \\
& \quad+3 T_{1} \int_{0}^{\xi_{T_{1}}} \int_{0}^{t_{1}}\left(\frac{\partial f_{n}}{\partial \tau_{2}}\right)^{2}\left(\tau, \tau+\xi-t_{1}\right) d \tau d t_{1} \\
& \quad \leq c\left\|f_{n}\right\|_{H^{1}(\omega)}^{2},
\end{aligned}
$$




$$
\begin{aligned}
& \left\|\frac{d g_{2 n}\left(t_{1}, \xi\right)}{d t_{1}}\right\|_{L^{2}\left(\xi, T_{1}\right)}^{2} \\
& \quad \leq \xi \int_{\xi}^{T_{1}} \int_{0}^{\xi}\left(\frac{\partial f_{n}}{\partial \tau_{1}}\right)^{2}\left(\tau+t_{1}-\xi, \tau\right) d \tau d t_{1} \\
& \quad \leq \xi\left\|f_{n}\right\|_{H^{1}(\omega)}^{2} \text { for } T_{1}>\xi .
\end{aligned}
$$

Hence, $g_{1 n}\left(t_{1}, \xi\right) \in H^{1}\left(0, \xi_{T_{1}}\right)$ and $g_{2 n}\left(t_{1}, \xi\right) \in H^{1}\left(\xi, T_{1}\right)$ for $T_{1}>\xi$, and from embedding theorem we obtain that $g_{1 n}\left(t_{1}, \xi\right) \in C\left[0, \xi_{T_{1}}\right], g_{2 n}\left(t_{1}, \xi\right) \in C\left[\xi, T_{1}\right]$ for $T_{1}>\xi$.

Note that the classical problem for (45) with initial conditions $u_{n}\left(0, t_{2}\right)=\varphi_{n}\left(t_{2}\right)$ and $u_{n}\left(t_{1}, 0\right)=d_{n}\left(t_{1}\right), d_{n} \in$ $H^{1}\left(0, T_{1}\right), \varphi_{n}(0)=d_{n}(0)$, has a unique solution $u_{n} \in$ $H^{1}(\omega), d u_{n}\left(t_{1}, 0\right) / d t_{1} \in L^{2}\left(0, T_{1}\right), d u_{n}\left(0, t_{2}\right) / d t_{2} \in L^{2}\left(0, T_{2}\right)$, which is given by

$$
\begin{aligned}
& u_{n}\left(t_{1}, t_{2}\right) \\
& \quad= \begin{cases}e^{-\lambda_{n}^{2} t_{1}} \varphi_{n}\left(t_{2}-t_{1}\right)+g_{1 n}\left(t_{1}, t_{2}\right), & \left(t_{1}, t_{2}\right) \in \omega_{1}, \\
e^{-\lambda_{n}^{2} t_{2}} d_{n}\left(t_{1}-t_{2}\right)+g_{2 n}\left(t_{1}, t_{2}\right), & \left(t_{1}, t_{2}\right) \in \omega_{2} .\end{cases}
\end{aligned}
$$

From nonlocal conditions (46) for function $d_{n}$ we have

$$
\begin{aligned}
& d_{n}\left(t_{1}\right) \\
& = \begin{cases}\alpha\left(e^{-\lambda_{n}^{2} t_{1}} \varphi_{n}\left(\xi-t_{1}\right)+g_{1 n}\left(t_{1}, \xi\right)\right)+\psi_{n}\left(t_{1}\right), & t_{1} \in\left[0, \xi_{T_{1}}\right], \\
\alpha\left(e^{-\lambda_{n}^{2} \xi} d_{n}\left(t_{1}-\xi\right)+g_{2 n}\left(t_{1}, \xi\right)\right)+\psi_{n}\left(t_{1}\right), & t_{1} \in\left(\xi_{T_{1}}, T_{1}\right],\end{cases}
\end{aligned}
$$

from which we obtain expression of $d_{n}$ for $t_{1}>\xi, T_{1}>\xi$,

$$
\begin{aligned}
& d_{n}\left(t_{1}\right) \\
& =\left(\alpha e^{-\lambda_{n}^{2} \xi}\right)^{k} d_{n}\left(t_{1}-k \xi\right) \\
& \quad+\sum_{i=0}^{k-1}\left(\alpha e^{-\lambda_{n}^{2} \xi}\right)^{i}\left(\alpha g_{2 n}\left(t_{1}-i \xi, \xi\right)+\psi_{n}\left(t_{1}-i \xi\right)\right)
\end{aligned}
$$

for $k \xi<t_{1} \leq \min \left\{(k+1) \xi, T_{1}\right\}, k=1, \ldots, K$, where $K=$ $\left[T_{1} / \xi\right]$, if $T_{1} / \xi \notin \mathbf{N}$, and $K=T_{1} / \xi-1$, if $T_{1} / \xi \in \mathbf{N} ;[x]$ denotes the integer part of $x \in \mathbf{R}$.

Since functions $\varphi_{n}, \psi_{n}, g_{1 n}\left(t_{1}, \xi\right)$, and $g_{2 n}\left(t_{1}, \xi\right)$ are continuous, we have $d_{n} \in C\left[0, \xi_{T_{1}}\right]$. Let us assume that $T_{1}>\xi$ and then $\xi_{T_{1}}=\xi$. Note that $g_{1 n}(0, \xi)=0, g_{1 n}(\xi, \xi)=g_{2 n}(\xi, \xi)$ and from compatibility condition we have $\varphi_{n}(0)=\alpha \varphi_{n}(\xi)+\psi_{n}(0)$. Hence, we get

$$
\begin{aligned}
d_{n}(\xi)= & \alpha\left(e^{-\lambda_{n}^{2} \xi} \varphi_{n}(0)+g_{1 n}(\xi, \xi)\right)+\psi_{n}(\xi) \\
= & \alpha\left(e^{-\lambda_{n}^{2} \xi}\left(\alpha \varphi_{n}(\xi)+\psi_{n}(0)\right)+g_{2 n}(\xi, \xi)\right) \\
& +\psi_{n}(\xi)
\end{aligned}
$$

$$
=\alpha\left(e^{-\lambda_{n}^{2} \xi} d_{n}(0)+g_{2 n}(\xi, \xi)\right)+\psi_{n}(\xi)
$$

which imply continuity of $d_{n}$ at point $\xi$. Moreover, we have

$$
\begin{aligned}
& d_{n}((k+1) \xi)=\left(\alpha e^{-\lambda_{n}^{2} \xi}\right)^{k} d_{n}(\xi)+\sum_{i=0}^{k-1}\left(\alpha e^{-\lambda_{n}^{2} \xi}\right)^{i} \\
& \cdot\left(\alpha g_{2 n}((k-i+1) \xi, \xi)+\psi_{n}((k-i+1) \xi)\right) \\
& =\left(\alpha e^{-\lambda_{n}^{2} \xi}\right)^{k+1} d_{n}(0)+\left(\alpha e^{-\lambda_{n}^{2} \xi}\right)^{k}\left(\alpha g_{2 n}(\xi, \xi)\right. \\
& \left.+\psi_{n}(\xi)\right)+\sum_{i=0}^{k-1}\left(\alpha e^{-\lambda_{n}^{2} \xi}\right)^{i}\left(\alpha g_{2 n}((k-i+1) \xi, \xi)\right. \\
& \left.+\psi_{n}((k-i+1) \xi)\right)=\left(\alpha e^{-\lambda_{n}^{2} \xi}\right)^{k+1} d_{n}(0) \\
& +\sum_{i=0}^{k}\left(\alpha e^{-\lambda_{n}^{2} \xi}\right)^{i} \\
& \quad\left(\alpha g_{2 n}((k-i+1) \xi, \xi)+\psi_{n}((k-i+1) \xi)\right) .
\end{aligned}
$$

Consequently, $d_{n} \in C\left[0, T_{1}\right]$ and its generalized derivative $d_{n}^{\prime}$ belongs to $L^{2}\left(0, T_{1}\right)$, since

$$
\begin{aligned}
& d_{n}^{\prime}\left(t_{1}\right)=\alpha\left(-\lambda_{n}^{2} e^{-\lambda_{n}^{2} t_{1}} \varphi_{n}\left(\xi-t_{1}\right)-e^{-\lambda_{n}^{2} t_{1}} \varphi_{n}^{\prime}\left(\xi-t_{1}\right)\right. \\
& \left.+\frac{d g_{1 n}\left(t_{1}, \xi\right)}{d t_{1}}\right)+\psi_{n}^{\prime}\left(t_{1}\right)
\end{aligned}
$$

for almost all $t_{1} \in\left(0, \xi_{T_{1}}\right)$ and

$$
\begin{aligned}
& d_{n}^{\prime}\left(t_{1}\right)=\left(\alpha e^{-\lambda_{n}^{2} \xi}\right)^{k} d_{n}^{\prime}\left(t_{1}-k \xi\right) \\
& \quad+\sum_{i=0}^{k-1}\left(\alpha e^{-\lambda_{n}^{2} \xi}\right)^{i}\left(\alpha \frac{d g_{2 n}\left(t_{1}-i \xi, \xi\right)}{d t_{1}}+\psi_{n}^{\prime}\left(t_{1}-i \xi\right)\right)
\end{aligned}
$$

for $T_{1}>\xi$ and for almost all $t_{1} \in\left(k \xi, \min \left\{(k+1) \xi, T_{1}\right\}\right), k=$ $1, \ldots, K$.

Since $g_{1 n}\left(0, t_{2}\right)=0$ and $g_{1 n}\left(0, t_{2}\right)=0$, from construction of $d_{n}$ it follows that $u_{n}$ satisfies conditions (46).

Note that the trace on the bisector $l_{T T}, T=\min \left\{T_{1}, T_{2}\right\}$, of restriction of function $u_{n}$ on $\omega_{1}$ equals the trace on the bisector $l_{T T}$ of restriction of function $u_{n}$ on $\omega_{2}$, because $g_{1 n}(t, t)=g_{2 n}(t, t), 0<t \leq T, \varphi_{n}(0)=\alpha \varphi_{n}(\xi)+\psi_{n}(0)$, and

$$
\begin{aligned}
u_{n}(t, t) & =e^{-\lambda_{n}^{2} t} \varphi_{n}(0)+g_{1 n}(t, t) \\
& =e^{-\lambda_{n}^{2} t}\left(\alpha \varphi_{n}(\xi)+\psi_{n}(0)\right)+g_{2 n}(t, t) \\
& =e^{-\lambda_{n}^{2} t} d_{n}(0)+g_{2 n}(t, t) .
\end{aligned}
$$


The generalized derivatives of function $u_{n}$ are given by

$$
\begin{aligned}
& \frac{\partial u_{n}}{\partial t_{1}}= \begin{cases}-\lambda_{n}^{2} e^{-\lambda_{n}^{2} t_{1}} \varphi_{n}\left(t_{2}-t_{1}\right)-e^{-\lambda_{n}^{2} t_{1}} \varphi_{n}^{\prime}\left(t_{2}-t_{1}\right)+\frac{\partial g_{1 n}}{\partial t_{1}}\left(t_{1}, t_{2}\right), & \left(t_{1}, t_{2}\right) \in \omega_{1}, \\
e^{-\lambda_{n}^{2} t_{2}} d_{n}^{\prime}\left(t_{1}-t_{2}\right)+\frac{\partial g_{2 n}}{\partial t_{1}}\left(t_{1}, t_{2}\right), & \left(t_{1}, t_{2}\right) \in \omega_{2},\end{cases} \\
& \frac{\partial u_{n}}{\partial t_{2}}= \begin{cases}e^{-\lambda_{n}^{2} t_{1}} \varphi_{n}^{\prime}\left(t_{2}-t_{1}\right)+\frac{\partial g_{1 n}}{\partial t_{2}}\left(t_{1}, t_{2}\right), \\
-\lambda_{n}^{2} e^{-\lambda_{n}^{2} t_{2}} d_{n}\left(t_{1}-t_{2}\right)-e^{-\lambda_{n}^{2} t_{2}} d_{n}^{\prime}\left(t_{1}-t_{2}\right)+\frac{\partial g_{2 n}}{\partial t_{2}}\left(t_{1}, t_{2}\right), & \left.\left(t_{1}, t_{2}\right) \in \omega_{1}\right)\end{cases}
\end{aligned}
$$

Therefore, we have $\partial u_{n} / \partial t_{1}, \partial u_{n} / \partial t_{2} \in L^{2}(\omega)$ and $u_{n}$ satisfies (45) in $L^{2}(\omega)$.

Now we obtain estimates for $u_{n}$, which permit one to prove the convergence of the sequence of approximate solutions $\left(w_{N}\right)_{N \geq 1}$.

Applying Lemmas 9 and 10 we obtain

$$
\begin{aligned}
\left\|u_{n}\right\|_{L^{2}(\omega)}^{2} \leq & 2 \int_{\omega_{1}} e^{-2 \lambda_{n}^{2} t_{1}} \varphi_{n}^{2}\left(t_{2}-t_{1}\right) d t_{1} d t_{2} \\
& +2 \int_{\omega_{1}} g_{1 n}^{2}\left(t_{1}, t_{2}\right) d t_{1} d t_{2} \\
& +2 \int_{\omega_{2}} e^{-2 \lambda_{n}^{2} t_{2}} d_{n}^{2}\left(t_{1}-t_{2}\right) d t_{1} d t_{2} \\
& +2 \int_{\omega_{2}} g_{2 n}^{2}\left(t_{1}, t_{2}\right) d t_{1} d t_{2} \\
\leq & \frac{1}{\lambda_{n}^{2}}\left\|\varphi_{n}\right\|_{L^{2}\left(0, T_{2}\right)}^{2}+\frac{c}{\lambda_{n}^{4}}\left\|f_{n}\right\|_{H^{1}(\omega)}^{2} \\
& +\frac{1}{\lambda_{n}^{2}}\left\|d_{n}\right\|_{L^{2}\left(0, T_{1}\right)}^{2} .
\end{aligned}
$$

To obtain estimate for $d_{n}$ without loss of generality we assume that $T_{1}>\xi$. Applying Lemmas 9-11 we get

$$
\begin{aligned}
& \left\|d_{n}\right\|_{L^{2}\left(0, T_{1}\right)}^{2}=\left\|d_{n}\right\|_{L^{2}(0, \xi)}^{2} \\
& \quad+\sum_{k=1}^{K} \int_{k \xi}^{\min \left\{(k+1) \xi, T_{1}\right\}}\left(\left(\alpha e^{-\lambda_{n}^{2} \xi}\right)^{k} d_{n}\left(t_{1}-k \xi\right)\right. \\
& \left.\quad+\sum_{i=0}^{k-1}\left(\alpha e^{-\lambda_{n}^{2} \xi}\right)^{i}\left(\alpha g_{2 n}\left(t_{1}-i \xi, \xi\right)+\psi_{n}\left(t_{1}-i \xi\right)\right)\right)^{2} d t_{1} \\
& \leq \int_{0}^{\xi} d_{n}^{2}\left(t_{1}\right) d t_{1}+K(2 K+1) \max \left\{\alpha^{2 K}, \alpha^{2}\right\} \\
& \quad \cdot \int_{0}^{\xi} d_{n}^{2}(s) d s+K(2 K+1) \max \left\{\alpha^{2 K}, \alpha^{2}\right\} \int_{\xi}^{T_{1}} g_{2 n}^{2}\left(t_{1},\right. \\
& \xi) d t_{1}+K(2 K+1) \max \left\{1, \alpha^{2(K-1)}\right\} \int_{\xi}^{T_{1}} \psi_{n}^{2}(s) d s
\end{aligned}
$$

$$
\begin{aligned}
& \leq\left(1+K(2 K+1) \max \left\{\alpha^{2 K}, \alpha^{2}\right\}\right)\left(3 \alpha^{2} \int_{0}^{\xi} \varphi_{n}^{2}(\xi\right. \\
& \left.-t_{1}\right) d t_{1}+3 \alpha^{2} \int_{0}^{\xi} g_{1 n}^{2}\left(t_{1}, \xi\right) d t_{1} \\
& \left.+3 \int_{0}^{\xi} \psi_{n}^{2}\left(t_{1}\right) d t_{1}\right)+K(2 K+1) \max \left\{\alpha^{2 K}, \alpha^{2}\right\} \\
& \cdot \frac{c}{\lambda_{n}^{4}}\left\|f_{n}\right\|_{H^{1}(\omega)}^{2}+K(2 K+1) \max \left\{1, \alpha^{2(K-1)}\right\} \\
& \cdot \int_{\xi}^{T_{1}} \psi_{n}^{2}(s) d s \leq c\left(\left\|\varphi_{n}\right\|_{L^{2}\left(0, T_{2}\right)}^{2}+\left\|\psi_{n}\right\|_{L^{2}\left(0, T_{1}\right)}^{2}\right. \\
& \left.+\frac{1}{\lambda_{n}^{4}}\left\|f_{n}\right\|_{H^{1}(\omega)}^{2}\right) .
\end{aligned}
$$

From the latter estimate we have

$\left\|u_{n}\right\|_{L^{2}(\omega)}^{2}$

$$
\leq c\left(\frac{\left\|\varphi_{n}\right\|_{L^{2}\left(0, T_{2}\right)}^{2}}{\lambda_{n}^{2}}+\frac{\left\|\psi_{n}\right\|_{L^{2}\left(0, T_{1}\right)}^{2}}{\lambda_{n}^{2}}+\frac{\left\|f_{n}\right\|_{H^{1}(\omega)}^{2}}{\lambda_{n}^{4}}\right) .
$$

Applying expressions for $\partial u_{n} / \partial t_{1}$ and $\partial u_{n} / \partial t_{2}$, Lemma 9, and estimates (51)-(54) we obtain

$$
\begin{aligned}
\left\|\frac{\partial u_{n}}{\partial t_{1}}\right\|_{L^{2}(\omega)}^{2} \leq & 3 \lambda_{n}^{4}\left\|e^{-\lambda_{n}^{2} t_{1}} \varphi_{n}\left(t_{2}-t_{1}\right)\right\|_{L^{2}\left(\omega_{1}\right)}^{2} \\
& +3\left\|e^{-\lambda_{n}^{2} t_{1}} \varphi_{n}^{\prime}\left(t_{2}-t_{1}\right)\right\|_{L^{2}\left(\omega_{1}\right)}^{2} \\
& +3\left\|\frac{\partial g_{1 n}}{\partial t_{1}}\right\|_{L^{2}\left(\omega_{1}\right)}^{2} \\
& +2\left\|e^{-\lambda_{n}^{2} t_{2}} d_{n}^{\prime}\left(t_{1}-t_{2}\right)\right\|_{L^{2}\left(\omega_{2}\right)}^{2} \\
& +2\left\|\frac{\partial g_{2 n}}{\partial t_{1}}\right\|_{L^{2}\left(\omega_{2}\right)}^{2}
\end{aligned}
$$




$$
\begin{aligned}
\leq & \frac{3 \lambda_{n}^{2}}{2}\left\|\varphi_{n}\right\|_{L^{2}\left(0, T_{2}\right)}^{2}+\frac{3}{2 \lambda_{n}^{2}}\left\|\varphi_{n}^{\prime}\right\|_{L^{2}\left(0, T_{2}\right)}^{2} \\
& +\frac{1}{\lambda_{n}^{2}}\left\|d_{n}^{\prime}\right\|_{L^{2}\left(0, T_{1}\right)}^{2}+5 c\left\|f_{n}\right\|_{H^{1}(\omega)}^{2} .
\end{aligned}
$$

In order to estimate $d_{n}^{\prime}$ without loss of generality we assume that $T_{1}>\xi$. Applying Lemmas 9-11 we have

$$
\begin{aligned}
& \left\|d_{n}^{\prime}\right\|_{L^{2}\left(0, T_{1}\right)}^{2}=\left\|d_{n}^{\prime}\right\|_{L^{2}(0, \xi)}^{2}+\sum_{k=1}^{K} \int_{k \xi}^{\min \left\{(k+1) \xi, T_{1}\right\}}\left(\left(\alpha e^{-\lambda_{n}^{2} \xi}\right)^{k}\right. \\
& \cdot d_{n}^{\prime}\left(t_{1}-k \xi\right) \\
& \left.+\sum_{i=0}^{k-1}\left(\alpha e^{-\lambda_{n}^{2} \xi}\right)^{i}\left(\alpha \frac{\partial g_{2 n}}{\partial t_{1}}\left(t_{1}-i \xi, \xi\right)+\psi_{n}^{\prime}\left(t_{1}-i \xi\right)\right)\right)^{2} d t_{1} \\
& \leq\left\|d_{n}^{\prime}\right\|_{L^{2}(0, \xi)}^{2}+K(2 K+1) \max \left\{\alpha^{2 K}, \alpha^{2}\right\}\left\|d_{n}^{\prime}\right\|_{L^{2}(0, \xi)}^{2} \\
& +K(2 K+1) \max \left\{\alpha^{2 K}, \alpha^{2}\right\} \int_{\xi}^{T_{1}}\left(\frac{d g_{2 n}\left(t_{1}, \xi\right)}{d t_{1}}\right)^{2} d t_{1} \\
& +K(2 K+1) \max \left\{1, \alpha^{2(K-1)}\right\}\left\|\psi_{n}^{\prime}\right\|_{L^{2}\left(\xi, T_{1}\right)}^{2} \leq(1+K(2 K+1) \\
& \left.\cdot \max \left\{\alpha^{2 K}, \alpha^{2}\right\}\right)\left(4 \alpha^{2} \lambda_{n}^{4} \int_{0}^{\xi} \varphi_{n}^{2}\left(\xi-t_{1}\right) d t_{1}\right. \\
& +4 \alpha^{2} \int_{0}^{\xi}\left(\varphi_{n}^{\prime}\left(\xi-t_{1}\right)\right)^{2} d t_{1}+4 \alpha^{2} \int_{0}^{\xi}\left(\frac{d g_{1 n}\left(t_{1}, \xi\right)}{d t_{1}}\right)^{2} d t_{1} \\
& \left.+4 \int_{0}^{\xi}\left(\psi_{n}^{\prime}\right)^{2}\left(t_{1}\right) d t_{1}\right)+K(2 K+1) \max \left\{\alpha^{2 K}, \alpha^{2}\right\} \\
& +\xi\left\|f_{n}\right\|_{H^{1}(\omega)}^{2}+K(2 K+1) \max \left\{1, \alpha^{2(K-1)}\right\}\left\|\psi_{n}^{\prime}\right\|_{L^{2}\left(\xi, T_{1}\right)}^{2} \\
& \leq c\left(\lambda_{n}^{4}\left\|\varphi_{n}\right\|_{L^{2}\left(0, T_{2}\right)}^{2}+\left\|\varphi_{n}^{\prime}\right\|_{L^{2}\left(0, T_{2}\right)}^{2}+\left\|\psi_{n}^{\prime}\right\|_{L^{2}\left(0, T_{1}\right)}^{2}+\left\|f_{n}\right\|_{H^{1}(\omega)}^{2}\right)
\end{aligned}
$$

From the estimate for $\left\|\partial u_{n} / \partial t_{1}\right\|_{L^{2}(\omega)}^{2}$ and (69) we get

$$
\begin{gathered}
\left\|\frac{\partial u_{n}}{\partial t_{1}}\right\|_{L^{2}(\omega)}^{2} \leq c\left(\lambda_{n}^{2}\left\|\varphi_{n}\right\|_{L^{2}\left(0, T_{2}\right)}^{2}+\frac{1}{\lambda_{n}^{2}}\left\|\varphi_{n}^{\prime}\right\|_{L^{2}\left(0, T_{2}\right)}^{2}\right. \\
\left.+\frac{1}{\lambda_{n}^{2}}\left\|\psi_{n}^{\prime}\right\|_{L^{2}\left(0, T_{1}\right)}^{2}+\left\|f_{n}\right\|_{H^{1}(\omega)}^{2}\right) .
\end{gathered}
$$

By using Lemma 9 and estimates (52), (54), (66), and (69) we obtain

$$
\begin{aligned}
& \left\|\frac{\partial u_{n}}{\partial t_{2}}\right\|_{L^{2}(\omega)}^{2} \leq 2\left\|e^{-\lambda_{n}^{2} t_{1}} \varphi_{n}^{\prime}\left(t_{2}-t_{1}\right)\right\|_{L^{2}\left(\omega_{1}\right)}^{2} \\
& +2\left\|\frac{\partial g_{1 n}}{\partial t_{2}}\right\|_{L^{2}\left(\omega_{1}\right)}^{2}+3 \lambda_{n}^{4}\left\|e^{-\lambda_{n}^{2} t_{2}} d_{n}\left(t_{1}-t_{2}\right)\right\|_{L^{2}\left(\omega_{2}\right)}^{2} \\
& +3\left\|e^{-\lambda_{n}^{2} t_{2}} d_{n}^{\prime}\left(t_{1}-t_{2}\right)\right\|_{L^{2}\left(\omega_{2}\right)}^{2}+3\left\|\frac{\partial g_{2 n}}{\partial t_{2}}\right\|_{L^{2}\left(\omega_{2}\right)}^{2} \\
& \leq \frac{1}{\lambda_{n}^{2}}\left\|\varphi_{n}^{\prime}\right\|_{L^{2}\left(0, T_{2}\right)}^{2}+c\left\|f_{n}\right\|_{H^{1}(\omega)}^{2}+\frac{3 \lambda_{n}^{2}}{2}\left\|d_{n}\right\|_{L^{2}\left(0, T_{1}\right)}^{2}
\end{aligned}
$$

$$
\begin{aligned}
& +\frac{3\left\|d_{n}^{\prime}\right\|_{L^{2}\left(0, T_{1}\right)}^{2}}{2 \lambda_{n}^{2}} \leq c\left(\lambda_{n}^{2}\left\|\varphi_{n}\right\|_{L^{2}\left(0, T_{2}\right)}^{2}+\frac{\left\|\varphi_{n}^{\prime}\right\|_{L^{2}\left(0, T_{2}\right)}^{2}}{\lambda_{n}^{2}}\right. \\
& \left.+\lambda_{n}^{2}\left\|\psi_{n}\right\|_{L^{2}\left(0, T_{1}\right)}^{2}+\frac{1}{\lambda_{n}^{2}}\left\|\psi_{n}^{\prime}\right\|_{L^{2}\left(0, T_{1}\right)}^{2}+\left\|f_{n}\right\|_{H^{1}(\omega)}^{2}\right) .
\end{aligned}
$$

Applying parts (a) of Lemmas 4, 6, and 8 and inequalities (67), (70), and (71) we obtain

$$
\begin{aligned}
& \sum_{n=1}^{\infty} \lambda_{n}^{2}\left\|u_{n}\right\|_{L^{2}(\omega)}^{2} \leq c\left(\|\varphi\|_{L^{2}\left(0, T_{2} ; H\right)}^{2}+\|\psi\|_{L^{2}\left(0, T_{1}: H\right)}^{2}\right. \\
& \left.+\|f\|_{L^{2}\left(\omega: V^{\prime}\right)}^{2}\right), \\
& \sum_{n=1}^{\infty} \frac{1}{\lambda_{n}^{2}}\left\|\frac{\partial u_{n}}{\partial t_{i}}\right\|_{L^{2}(\omega)}^{2} \leq c\left(\|\varphi\|_{L^{2}\left(0, T_{2} ; H\right)}^{2}+\left\|\varphi^{\prime}\right\|_{L^{2}\left(0, T_{2} ; V_{1}^{\prime}\right)}^{2}\right. \\
& \left.+\|\psi\|_{L^{2}\left(0, T_{1}: H\right)}^{2}+\left\|\psi^{\prime}\right\|_{L^{2}\left(0, T_{1}: V_{1}^{\prime}\right)}^{2}+\|f\|_{L^{2}\left(\omega ; V^{\prime}\right)}\right), \quad i=1,2 . \\
& \left.+\left\|\frac{\partial f}{\partial t_{1}}\right\|_{L^{2}\left(\omega ; V^{\prime}\right)}+\left\|\frac{\partial f}{\partial t_{2}}\right\|_{L^{2}\left(\omega ; V^{\prime}\right)}\right), \quad
\end{aligned}
$$

Consequently, from parts (b) of Lemmas 5 and 6 we get the sequence $\left(w_{N}\right)_{N \geq 1}$ and hence the series

$$
u\left(t_{1}, t_{2}\right)=\sum_{n=1}^{\infty} u_{n}\left(t_{1}, t_{2}\right) v_{n}
$$

converges in the space $W$. Since the trace operators $\operatorname{tr}_{t_{1}=0}$, $\operatorname{tr}_{t_{2}=0}$, and $\operatorname{tr}_{t_{2}=\xi}$ are continuous, from (46) we obtain that $u$ satisfies conditions (35), and, by (72), the estimate (44) is valid. Moreover,

$$
\begin{aligned}
\operatorname{tr}_{t_{1}=0} u & =\lim _{N \rightarrow \infty} \sum_{n=1}^{N} u_{n}\left(0, t_{2}\right) v_{n} \in L^{2}\left(0, T_{2} ; H\right) \\
& \subset D^{\prime}\left(0, T_{2} ; H\right), \\
\operatorname{tr}_{t_{2}=0} u & =\lim _{N \rightarrow \infty} \sum_{n=1}^{N} u_{n}\left(t_{1}, 0\right) v_{n} \in L^{2}\left(0, T_{1} ; H\right) \\
& \subset D^{\prime}\left(0, T_{1} ; H\right),
\end{aligned}
$$

and since the operator of differentiation is continuous on the space of distributions we have

$$
\begin{aligned}
\frac{d\left(\operatorname{tr}_{t_{1}=0} u\right)}{d t_{2}} & =\lim _{N \rightarrow \infty} \sum_{n=1}^{N} \frac{d\left(u_{n}\left(0, t_{2}\right)\right)}{d t_{2}} v_{n} \\
& \in D^{\prime}\left(0, T_{2} ; H\right), \\
\frac{d\left(\operatorname{tr}_{t_{2}=0} u\right)}{d t_{1}} & =\lim _{N \rightarrow \infty} \sum_{n=1}^{N} \frac{d\left(u_{n}\left(t_{1}, 0\right)\right)}{d t_{1}} v_{n} \\
& \in D^{\prime}\left(0, T_{1} ; H\right) .
\end{aligned}
$$


Applying part (a) of Lemma 8 we obtain

$$
\begin{aligned}
& \sum_{n=1}^{N} \frac{1}{\lambda_{n}^{4}}\left\|\frac{d\left(u_{n}\left(0, t_{2}\right)\right)}{d t_{2}}\right\|_{L^{2}\left(0, T_{2}\right)}^{2}=\sum_{n=1}^{N} \frac{\left\|\varphi_{n}^{\prime}\right\|_{L^{2}\left(0, T_{2}\right)}^{2}}{\lambda_{n}^{4}} \\
& \quad \leq\left\|\left(A_{1} A\right)^{-1}\right\|^{2}\left\|\varphi^{\prime}\right\|_{L^{2}\left(0, T_{2} ; V_{1}^{\prime}\right)}^{2},
\end{aligned}
$$

and by using parts (a) of Lemmas 4-6 and (69) we get

$$
\begin{aligned}
& \sum_{n=1}^{N} \frac{1}{\lambda_{n}^{4}}\left\|\frac{d\left(u_{n}\left(t_{1}, 0\right)\right)}{d t_{1}}\right\|_{L^{2}\left(0, T_{2}\right)}^{2}=\sum_{n=1}^{N} \frac{\left\|d_{n}^{\prime}\right\|_{L^{2}\left(0, T_{1}\right)}^{2}}{\lambda_{n}^{4}} \\
& \quad \leq c\left(\left\|\varphi_{n}\right\|_{L^{2}\left(0, T_{2}\right)}^{2}+\frac{\left\|\varphi_{n}^{\prime}\right\|_{L^{2}\left(0, T_{2}\right)}^{2}}{\lambda_{n}^{4}}+\frac{\left\|\psi_{n}^{\prime}\right\|_{L^{2}\left(0, T_{1}\right)}^{2}}{\lambda_{n}^{4}}\right. \\
& \left.+\frac{\left\|f_{n}\right\|_{H^{1}(\omega)}^{2}}{\lambda_{n}^{4}}\right) \leq c\left(\|\varphi\|_{L^{2}\left(0, T_{2} ; H\right)}^{2}+\left\|\varphi^{\prime}\right\|_{L^{2}\left(0, T_{2} ; V_{1}^{\prime}\right)}^{2}\right. \\
& +\left\|\psi^{\prime}\right\|_{L^{2}\left(0, T_{1}: V_{1}^{\prime}\right)}^{2}+\|f\|_{L^{2}\left(\omega ; V^{\prime}\right)}+\left\|\frac{\partial f}{\partial t_{1}}\right\|_{L^{2}\left(\omega ; V^{\prime}\right)} \\
& \left.+\left\|\frac{\partial f}{\partial t_{2}}\right\|_{L^{2}\left(\omega ; V^{\prime}\right)}\right) .
\end{aligned}
$$

Consequently, from part (b) of Lemma 8 we obtain

$$
\begin{aligned}
\frac{d\left(\operatorname{tr}_{t_{1}=0} u\right)}{d t_{2}} & =\lim _{N \rightarrow \infty} \sum_{n=1}^{N} \frac{d\left(u_{n}\left(0, t_{2}\right)\right)}{d t_{2}} v_{n} \\
& \in L^{2}\left(0, T_{2} ; V_{1}^{\prime}\right) \\
\frac{d\left(\operatorname{tr}_{t_{2}=0} u\right)}{d t_{1}} & =\lim _{N \rightarrow \infty} \sum_{n=1}^{N} \frac{d\left(u_{n}\left(t_{1}, 0\right)\right)}{d t_{1}} v_{n} \\
& \in L^{2}\left(0, T_{1} ; V_{1}^{\prime}\right) .
\end{aligned}
$$

Let us show that $u \in W, d\left(\operatorname{tr}_{t_{2}=0} u\right) / d t_{1} \in L^{2}\left(0, T_{1} ; V_{1}^{\prime}\right)$, $d\left(\operatorname{tr}_{t_{1}=0} u\right) / d t_{2} \in L^{2}\left(0, T_{2} ; V_{1}^{\prime}\right)$ is a solution of (34). Indeed, since the system $\left\{v_{k}\right\}_{k \in \mathbf{N}}$ is complete in $V$, it follows that for each $v \in V$ there exists a sequence $\left(z_{k}\right)_{k \geq 1}$, where $z_{k}$ is a linear combination of $v_{1}, \ldots, v_{k}$, such that $z_{k} \rightarrow v$ in $V$ as $k \rightarrow \infty$. By taking account of the properties of eigenvectors $\left\{v_{k}\right\}_{k \in \mathbf{N}}$, from (45) for each $\psi \in D(\omega)$ and $v \in V$ we have

$$
\begin{gathered}
-\int_{\omega}\left(w_{N}, z_{N}\right) \frac{\partial \psi}{\partial t_{1}} d t_{1} d t_{2}-\int_{\omega}\left(w_{N}, z_{N}\right) \frac{\partial \psi}{\partial t_{2}} d t_{1} d t_{2} \\
+\int_{\omega} a\left(w_{N}, z_{N}\right) \psi d t_{1} d t_{2}=0, \quad \forall N \in \mathbf{N} .
\end{gathered}
$$

By passing to the limit in the last relation, we find that $u$ satisfies (34). So, the existence of solution is proved.
To complete the proof, we prove the uniqueness of solution. Note that the classical problem for (34) with initial conditions

$$
\begin{array}{ll}
\left(\operatorname{tr}_{t_{1}=0} u\right)\left(t_{2}\right)=\widetilde{\varphi}\left(t_{2}\right), & 0 \leq t_{2} \leq T_{2}, \\
\left(\operatorname{tr}_{t_{2}=0} u\right)\left(t_{1}\right)=\widetilde{\psi}\left(t_{1}\right), & 0 \leq t_{1} \leq T_{1},
\end{array}
$$

has at most one solution. Indeed, if $\widetilde{u}$ is a solution of the homogeneous classical problem (34), (80), that is, $\widetilde{\varphi} \equiv 0, \widetilde{\psi} \equiv$ 0 and $f \equiv 0$, then, by multiplying (1), which is equivalent to $(34)$, by $u$, integrating on $\left(0, T_{1}\right) \times\left(0, T_{2}\right)$, and applying Theorem 2 we get

$$
\begin{aligned}
\frac{1}{2} \int_{0}^{T_{2}} & \left\|\left(\operatorname{tr}_{t_{1}=T_{1}} \tilde{u}\right)\left(t_{2}\right)\right\|_{H}^{2} d t_{2} \\
& +\frac{1}{2} \int_{0}^{T_{1}}\left\|\left(\operatorname{tr}_{t_{2}=T_{2}} \tilde{u}\right)\left(t_{1}\right)\right\|_{H}^{2} d t_{1} \\
& +\int_{0}^{T_{1}} \int_{0}^{T_{2}} a(\tilde{u}, \tilde{u}) d t_{2} d t_{1}=0,
\end{aligned}
$$

and, consequently, $\widetilde{u} \equiv 0$ in $\left(0, T_{1}\right) \times\left(0, T_{2}\right)$.

Since problem (34), (35) for $\alpha=0$ is a classical problem, from the proof of the existence it follows that the classical problem for (34) with initial conditions (80), $\widetilde{\varphi} \in$ $L^{2}\left(0, T_{2} ; H\right), \widetilde{\varphi}^{\prime} \in L^{2}\left(0, T_{2} ; V_{1}^{\prime}\right), \widetilde{\psi} \in L^{2}\left(0, T_{1} ; H\right), \widetilde{\psi}^{\prime} \in$ $L^{2}\left(0, T_{1} ; V_{1}^{\prime}\right), \widetilde{\varphi}(0)=\widetilde{\psi}(0)$, has a unique solution $u \in W$, $\operatorname{tr}_{t_{1}=0} u \in L^{2}\left(0, T_{2} ; H\right), d\left(\operatorname{tr}_{t_{1}=0} u\right) / d t_{2} \in L^{2}\left(0, T_{2} ; V_{1}^{\prime}\right), \operatorname{tr}_{t_{2}=0} u$ $\in L^{2}\left(0, T_{1} ; H\right), d\left(\operatorname{tr}_{t_{2}=0} u\right) / d t_{1} \in L^{2}\left(0, T_{1} ; V_{1}^{\prime}\right)$, which can be represented in the form of convergent in $W$ series (73), where $u_{n}$ is given by (56). Therefore, the uniqueness of the solution of nonlocal problem (34), (35) follows from the uniqueness of the solution of problem (45), (46), which completes the proof.

\section{Application}

In this section, we consider an application of Theorem 12 obtained for abstract nonlocal problem to nonclassical problem for ultraparabolic partial differential equation with nonlocal initial condition. Let $\Omega \subset \mathbf{R}^{n}, n \in \mathbf{N}$, be a bounded domain with Lipschitz boundary $\Gamma$ [47]. By $H^{k}(\Omega)$ we denote the Sobolev space of functions in $L^{2}(\Omega)$ whose generalized partial derivatives up to the order $k \in \mathbf{N}$ belong to $L^{2}(\Omega)$. We denote the closure in $H^{k}(\Omega)$ of the set $D(\Omega)$ by $H_{0}^{k}(\Omega)$ and its dual space by $H^{-k}(\Omega)$. We consider the second-order elliptic operator:

$$
A \equiv-\sum_{q, j=1}^{n} \frac{\partial}{\partial x_{q}}\left(a_{q j}(x) \frac{\partial}{\partial x_{j}}\right)+a_{0}(x),
$$

where $a_{q j}(x), a_{0}(x) \in L^{\infty}(\Omega), q, j=1, \ldots, n$, and the following inequalities are valid

$$
a_{0}(x) \geq 0 \text {, }
$$

$$
\sum_{q, j=1}^{n} a_{q j}(x) \eta_{j} \eta_{q} \geq c_{A} \sum_{j=1}^{n}\left(\eta_{j}\right)^{2}, \quad c_{A}=\text { const }>0
$$


for almost all $x \in \Omega, \eta_{j}(j=1, \ldots, n)$ are arbitrary real numbers. Partial derivatives in the definition of $A$ are treated as generalized derivatives with respect to the corresponding variables. Note that the first-order partial derivatives of a function in $L^{2}(\Omega)$ belongs to the space $H^{-1}(\Omega)$. Since the multiplication of a function in the space $L^{2}(\Omega)$ by a function in $L^{\infty}(\Omega)$ leaves it in $L^{2}(\Omega)$, for each function $v \in H_{0}^{1}(\Omega)$ we have $A v \in H^{-1}(\Omega)$. The elliptic operator $A$ is a continuous operator from $H_{0}^{1}(\Omega)$ to the space $H^{-1}(\Omega)$, which, by virtue of (83) and Poincare inequality, satisfies the following selfadjointness and coerciveness conditions:

$$
\begin{aligned}
\langle A v, w\rangle_{*} & =\langle A w, v\rangle_{*}, \\
\langle A v, v\rangle_{*} & \geq \alpha\|v\|_{H^{1}(\Omega)}^{2},
\end{aligned}
$$

$$
\forall v, w \in H_{0}^{1}(\Omega),
$$

where $\alpha=$ const $>0$ and $\langle\cdot, \cdot\rangle_{*}$ is the duality relation between the spaces $H^{-1}(\Omega)$ and $H_{0}^{1}(\Omega)$. We denote by $D(A)=$ $\left\{v \in H_{0}^{1}(\Omega) ; A v \in L^{2}(\Omega)\right\}$ the domain of operator $A$ : $D(A) \rightarrow L^{2}(\Omega)$. The bilinear form $a(v, w)=\langle A v, w\rangle_{*}$ corresponding to the operator $A$ is of the form

$$
a(v, w)=\int_{\Omega}\left(\sum_{q, j=1}^{n} a_{q j}(x) \frac{\partial v}{\partial x_{j}} \frac{\partial w}{\partial x_{q}}+a_{0}(x) v w\right) d x
$$

and satisfies conditions (11). Since the continuous embedding of $H_{0}^{1}(\Omega)$ in $L^{2}(\Omega)$ is compact, it follows from general theorem for self-adjoint coercive operators mapping a Hilbert space into its dual [47] that there exists a system of eigenfunctions $\left\{v_{k}\right\}_{k=1}^{\infty}$ of the operator $A: H_{0}^{1}(\Omega) \rightarrow H^{-1}(\Omega)$ corresponding to the eigenvalues $\left\{\lambda_{k}^{2}\right\}_{k=1}^{\infty}$, such that $\left\{v_{k}\right\}_{k=1}^{\infty}$ is orthonormal in $L^{2}(\Omega)$ and complete in $H_{0}^{1}(\Omega)$, and $0<\lambda_{1} \leq$ $\lambda_{2} \leq \cdots, \lambda_{k} \rightarrow \infty$ as $k \rightarrow \infty$.

Let us consider nonclassical problem for ultraparabolic partial differential equation

$$
\frac{\partial u}{\partial t_{1}}+\frac{\partial u}{\partial t_{2}}+A u=f \quad \text { in } \Omega \times\left(0, T_{1}\right) \times\left(0, T_{2}\right),
$$

with nonlocal initial and homogeneous boundary conditions

$$
\begin{aligned}
u\left(x, 0, t_{2}\right) & =\varphi\left(x, t_{2}\right), \quad\left(x, t_{2}\right) \in \Omega \times\left(0, T_{2}\right), \\
u\left(x, t_{1}, 0\right) & =\alpha u\left(x, t_{1}, \xi\right)+\psi\left(x, t_{1}\right), \\
& \left(x, t_{1}\right) \in \Omega \times\left(0, T_{1}\right), \\
u\left(x, t_{1}, t_{2}\right) & =0, \quad\left(x, t_{1}, t_{2}\right) \in \Gamma \times\left(0, T_{1}\right) \times\left(0, T_{2}\right),
\end{aligned}
$$

where $\alpha \in \mathbf{R}$. We identify functions defined on $\Omega \times \omega$ with vector-functions defined on $\omega$ and ranging in the corresponding function spaces defined on $\Omega$. Hence, the nonlocal in time problem $(86)-(88)$ can be stated as the problem of finding a vector-function $u \in L^{2}\left(\omega ; H_{0}^{1}(\Omega)\right)$, $\partial u / \partial t_{1}$, $\partial u / \partial t_{2} \in L^{2}\left(\omega ; H^{-1}(\Omega)\right), d\left(\operatorname{tr}_{t_{2}=0} u\right) / d t_{1} \in L^{2}\left(0, T_{1} ;(D(A))^{\prime}\right)$, $d\left(\operatorname{tr}_{t_{1}=0} u\right) / d t_{2} \in L^{2}\left(0, T_{2} ;(D(A))^{\prime}\right)$, which satisfies (86) in the space $D^{\prime}\left(\omega ; H^{-1}(\Omega)\right)$ of distributions on $\omega$ ranging in $H^{-1}(\Omega)$, where the partial derivatives $\partial u / \partial t_{1}$ and $\partial u / \partial t_{2}$ are treated as the first-order generalized derivatives $\partial u / \partial t_{1}$, $\partial u / \partial t_{2} \in D^{\prime}\left(\omega ; H_{0}^{1}(\Omega)\right)$. The unknown function $u$ satisfies conditions (87) in the spaces $L^{2}\left(0, T_{2} ; L^{2}(\Omega)\right)$ and $L^{2}\left(0, T_{1}\right.$; $L^{2}(\Omega)$ ), respectively. The boundary conditions (88) are valid, because the trace of function from $H_{0}^{1}(\Omega)$ vanishes on the boundary of the domain $\Omega$. Thus, nonlocal in time problem (86) $-(88)$ is a particular case of the nonclassical problem (34), (35) for $V=H_{0}^{1}(\Omega), H=L^{2}(\Omega)$, and $V_{1}=D(A)$. The following existence and uniqueness theorem is valid for nonlocal in time problem (86)-(88).

Theorem 13. If $\varphi \in L^{2}\left(0, T_{2} ; L^{2}(\Omega)\right), \varphi^{\prime} \in L^{2}\left(0, T_{2} ;(D(A))^{\prime}\right)$, $\psi \in L^{2}\left(0, T_{1} ; L^{2}(\Omega)\right), \psi^{\prime} \in L^{2}\left(0, T_{1} ;(D(A))^{\prime}\right), \varphi$ and $\psi$ satisfy compatibility condition $\langle\varphi(0), v\rangle_{*}=\alpha\langle\varphi(\xi), v\rangle_{*}+\langle\psi(0), v\rangle_{*}$ for all $v \in H_{0}^{1}(\Omega)$ and $f, \partial f / \partial t_{1}, \partial f / \partial t_{2} \in L^{2}\left(\omega ; H^{-1}(\Omega)\right)$, then nonlocal problem (86)-(88) has a unique solution $u \in$ $L^{2}\left(\omega ; H_{0}^{1}(\Omega)\right), \partial u / \partial t_{1}, \partial u / \partial t_{2} \in L^{2}\left(\omega ; H^{-1}(\Omega)\right), d\left(\operatorname{tr}_{t_{2}=0} u\right) /$ $d t_{1} \in L^{2}\left(0, T_{1} ;(D(A))^{\prime}\right), d\left(\operatorname{tr}_{t_{1}=0} u\right) / d t_{2} \in L^{2}\left(0, T_{2} ;(D(A))^{\prime}\right)$, and the following estimate is valid:

$$
\begin{aligned}
& \|u\|_{L^{2}\left(\omega ; H_{0}^{1}(\Omega)\right)}^{2}+\sum_{i=1}^{2}\left\|\frac{\partial u}{\partial t_{i}}\right\|_{L^{2}\left(\omega ; H^{-1}(\Omega)\right)}^{2} \\
& \leq c\left(\|\varphi\|_{L^{2}\left(0, T_{2} ; L^{2}(\Omega)\right)}^{2}+\left\|\varphi^{\prime}\right\|_{L^{2}\left(0, T_{2} ;(D(A))^{\prime}\right)}^{2}\right. \\
& +\|\psi\|_{L^{2}\left(0, T_{1} ; L^{2}(\Omega)\right)}^{2}+\left\|\psi^{\prime}\right\|_{L^{2}\left(0, T_{1} ;(D(A))^{\prime}\right)}^{2} \\
& +\|f\|_{L^{2}\left(\omega ; H^{-1}(\Omega)\right)}^{2}+\left\|\frac{\partial f}{\partial t_{1}}\right\|_{L^{2}\left(\omega ; H^{-1}(\Omega)\right)}^{2} \\
& \left.+\left\|\frac{\partial f}{\partial t_{2}}\right\|_{L^{2}\left(\omega ; H^{-1}(\Omega)\right)}^{2}\right) .
\end{aligned}
$$

\section{Conclusions}

In this paper, we investigated the existence and uniqueness of solution of nonclassical problem for ultraparabolic equation with two time variables and nonlocal initial condition, which connect values of the unknown vector-function at the initial and some later moment of time. The considered problem is a generalization of the classical problem for ultraparabolic equation and the obtained results give new well-posedness result for the classical problem, as well as for nonlocal one, in suitable function spaces with minimal regularity that is necessary to define the traces on the boundary of the time domain. Note that applying the results obtained in this paper and the presented method of investigation one can study various initial-boundary value problems for ultraparabolic equations with several time variables and more general nonlocal initial conditions. The proof of the existence of solution of the nonclassical problem is constructive and can be used to obtain approximate solutions of the nonlocal problem. 


\section{Competing Interests}

The authors declare that there is no conflict of interests regarding the publication of this paper.

\section{References}

[1] A. M. Il'in and R. Z. Khas'minskii, "On equations of brownian motion," Theory of Probability and Its Applications, vol. 9, no. 3, pp. 421-444, 1964.

[2] S. Chandresekhar, "Stochastic problems in physics and astronomy," Reviews of Modern Physics, vol. 15, pp. 1-89, 1943.

[3] A. V. Karaushev, River Hydraulic, Hydrometeorological Edition, Leningrad, 1969 (Russian).

[4] H. Risken, The Fokker-Planck Equation. Methods of Solution and Applications, vol. 18 of Springer Series in Synergetics, Springer, Berlin, Germany, 1984.

[5] A. I. Kozhanov, "On the solvability of boundary value problems for quasilinear ultraparabolic equations in some mathematical models of the dynamics of biological systems," Journal of Applied and Industrial Mathematics, vol. 4, no. 4, pp. 512-525, 2010.

[6] G. F. Webb, "Population models structured by age, size, and spatial position," in Structured Population Models in Biology and Epidemiology, vol. 1936 of Lecture Notes in Mathematics, pp. 149, Springer, Berlin, Germany, 2008.

[7] M. Di Francesco and A. Pascucci, "A continuous dependence result for ultraparabolic equations in option pricing," Journal of Mathematical Analysis and Applications, vol. 336, no. 2, pp. 1026-1041, 2007.

[8] A. D. Rendall, "The characteristic initial value problem for the Einstein equations," in Nonlinear Hyperbolic Equations and Field Theory (Varenna, 1990), vol. 253 of Pitman Research Notes in Mathematics Series, pp. 154-163, Longman Scientific \& Technical, 1992.

[9] J. Uglum, "Quantum cosmology of $R \times S^{2} \times S^{1}$," Physical Review, vol. 46, no. 3, pp. 4365-4372, 1992.

[10] A. Ashyralyev and S. Yilmaz, "An approximation of ultraparabolic equations," Abstract and Applied Analysis, vol. 2012, Article ID 840621, 14 pages, 2012.

[11] A. Bouziani, "On the solvability of nonlocal pluriparabolic problems," Electronic Journal of Differential Equations, vol. 2001, no. 21, pp. 1-16, 2001.

[12] T. G. Genčev, "Ultraparabolic equations," Soviet Mathematics, Doklady, vol. 4, pp. 979-982, 1963.

[13] L. G. Gomboev, "Stability estimates for the solutions of a certain ultraparabolic equation," Siberian Mathematical Journal, vol. 29, no. 1, pp. 156-159, 1988.

[14] A. M. Il'in, "On a class of ultraparabolic equations," Soviet Mathematics, Doklady, vol. 5, pp. 1673-1676, 1964.

[15] V. A. Khoa, T. N. Huy, L. T. Lan, and N. T. Y. Ngoc, "A finite difference scheme for nonlinear ultra-parabolic equations," Applied Mathematics Letters, vol. 46, pp. 70-76, 2015.

[16] S. Mesloub, "On a nonlocal problem for a pluriparabolic equation," Acta Universitatis Szegediensis. Acta Scientiarum Mathematicarum, vol. 67, no. 1-2, pp. 203-219, 2001.

[17] N. S. Piskunov, "Boundary value problems for equations of elliptic-parabolic type," Matematicheskii. Sbornik, vol. 7(49), no. 3, pp. 385-424, 1940 (Russian).

[18] S. Polidoro, "On a class of ultraparabolic operators of Kolmogorov-Fokker-Planck type," Le Matematiche, vol. 49, no. 1, pp. 53-105, 1994.
[19] S. Polidoro and M. A. Ragusa, "Hölder regularity for solutions of ultraparabolic equations in divergence form," Potential Analysis, vol. 14, no. 4, pp. 341-350, 2001.

[20] S. Polidoro and M. A. Ragusa, "Harnack inequality for hypoelliptic ultraparabolic equations with a singular lower order term," Revista Matematica Iberoamericana, vol. 24, no. 3, pp. 1011-1046, 2008.

[21] N. Protsakh, "Inverse problem for an ultraparabolic equation," Tatra Mountains Mathematical Publications, vol. 54, pp. 133-151, 2013.

[22] M. A. Ragusa, "On weak solutions of ultraparabolic equations," Nonlinear Analysis: Theory, Methods and Applications, vol. 47, no. 1, pp. 503-511, 2001.

[23] J. I. Šatyro, "The first boundary value problem for a certain ultraparabolic equation," Differencial'nye Uravnenija, vol. 7, pp. 1089-1096, 1971 (Russian).

[24] A. S. Tersenov, "Ultraparabolic equations and unsteady heat transfer," Journal of Evolution Equations, vol. 5, no. 2, pp. 277289, 2005.

[25] S. A. Tersenov, "Well-posedness of boundary value problems for a certain ultraparabolic equation," Siberian Mathematical Journal, vol. 40, no. 6, pp. 1157-1169, 1999.

[26] L. R. Volevich and S. G. Gindikin, "The Cauchy problem for pluriparabolic differential equations, II," Mathematics of the USSR-Sbornik, vol. 7, pp. 205-226, 1970.

[27] J.-L. Lions, "Sur certaines équations aux dérivées partielles à coefficients opérateurs non bornés," Journal d'Analyse Mathématique, vol. 6, no. 1, pp. 333-355, 1958.

[28] L. Lorenzi, "An abstract ultraparabolic integrodifferential equation," Le Matematiche, vol. 53, no. 2, pp. 401-435, 1998.

[29] K. Ramdani, M. Tucsnak, and J. Valein, "Detectability and state estimation for linear age-structured population diffusion models," ESAIM: Mathematical Modelling and Numerical Analysis, 2016.

[30] C. Walker, "Some remarks on the asymptotic behavior of the semigroup associated with age-structured diffusive populations," Monatshefte für Mathematik, vol. 170, no. 3-4, pp. 481501,2013

[31] S. G. Krejn and G. I. Laptev, "Boundary value problems for an equation in Hilbert space," Soviet Mathematics, Doklady, vol. 3, pp. 1350-1354, 1962.

[32] A. A. Kerefov, "Nonlocal boundary-value problems for parabolic equations," Differential Equations, vol. 15, pp. 52-54, 1979.

[33] G. Avalishvili and M. Avalishvili, "On nonclassical problems for first-order evolution equations," Georgian Mathematical Journal, vol. 18, pp. 441-463, 2011.

[34] G. Avalishvili, M. Avalishvili, and B. Miara, "Nonclassical problems with nonlocal initial conditions for second-order evolution equations," Asymptotic Analysis, vol. 76, no. 3-4, pp. 171-192, 2012.

[35] A. Benrabah, F. Rebbani, and N. Boussetila, "A study of the multitime evolution equation with time-nonlocal conditions," Balkan Journal of Geometry and Its Applications, vol. 16, no. 2, pp. 13-24, 2011.

[36] D. Gordeziani, M. Avalishvili, and G. Avalishvili, "On the investigation of one nonclassical problem for Navier-Stokes equations," Applied Mathematics and Informatics, vol. 7, no. 2, pp. 66-77, 2002.

[37] D. G. Gordeziani and G. A. Avalishvili, "Time-nonlocal problems for Schrödinger type equations. I: problems in abstract spaces," Differential Equations, vol. 41, no. 5, pp. 703-711, 2005. 
[38] D. G. Gordeziani and G. A. Avalishvili, “Time-nonlocal problems for Schrödinger type equations: II. Results for specific problems," Differential Equations, vol. 41, no. 6, pp. 852-859, 2005.

[39] V. S. Il'kiv and B. I. Ptashnyk, "An ill-posed nonlocal two-point problem for systems of partial differential equations," Siberian Mathematical Journal, vol. 46, no. 1, pp. 94-102, 2005.

[40] G. M. Lieberman, "Nonlocal problems for quasilinear parabolic equations," in Nonlinear Problems in Mathematical Physics and Related Topics I, vol. 1, pp. 247-270, Kluwer Academic/Plenum, New York, NY, USA, 2002.

[41] R. Quintanilla, "A note on a non-standard problem for an equation with a delay term," Applied Mathematics and Computation, vol. 216, no. 9, pp. 2759-2765, 2010.

[42] M. A. Ragusa, "Cauchy-Dirichlet problem associated to divergence form parabolic equations," Communications in Contemporary Mathematics, vol. 6, no. 3, pp. 377-393, 2004.

[43] È. M. Saydamatov, "Well-posedness of general nonlocal nonhomogeneous boundary value problems for pseudodifferential equations with partial derivatives," Siberian Advances in Mathematics, vol. 17, no. 3, pp. 213-226, 2007.

[44] V. V. Shelukhin, "A non-local in time model for radionuclides propagation in Stokes fluid," Dinamika Sploshnoi Sredy, vol. 107, pp. 180-193, 1993.

[45] V. V. Shelukhin, "A problem nonlocal in time for the equations of the dynamics of a barotropic ocean," Siberian Mathematical Journal, vol. 36, no. 3, pp. 608-630, 1995.

[46] R. Dautray and J.-L. Lions, Mathematical Analysis and Numerical Methods for Science and Technology, Volume 5: Evolution Problems I, Springer, Berlin, Germany, 2000.

[47] W. McLean, Strongly Elliptic Systems and Boundary Integral Equations, Cambridge University Press, Cambridge, UK, 2000. 


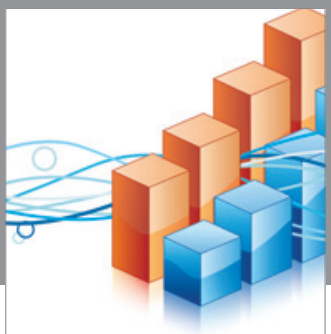

Advances in

Operations Research

vatem alat4

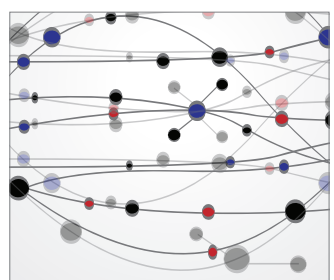

\section{The Scientific} World Journal
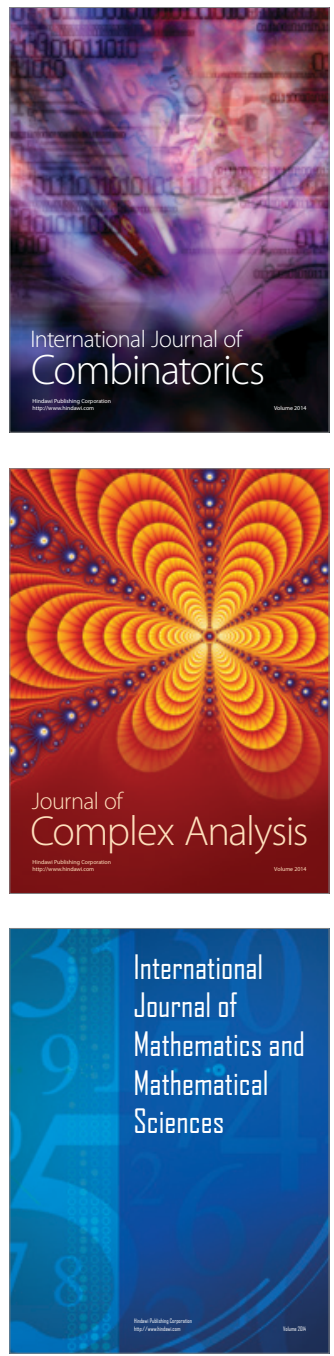
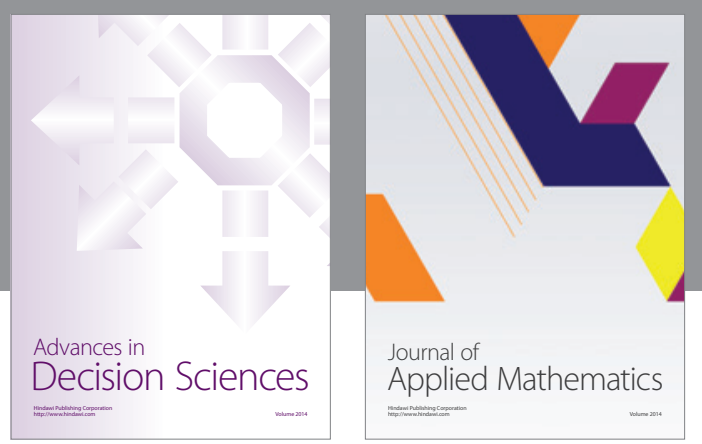

Algebra



\section{Hindawi}

Submit your manuscripts at

http://www.hindawi.com
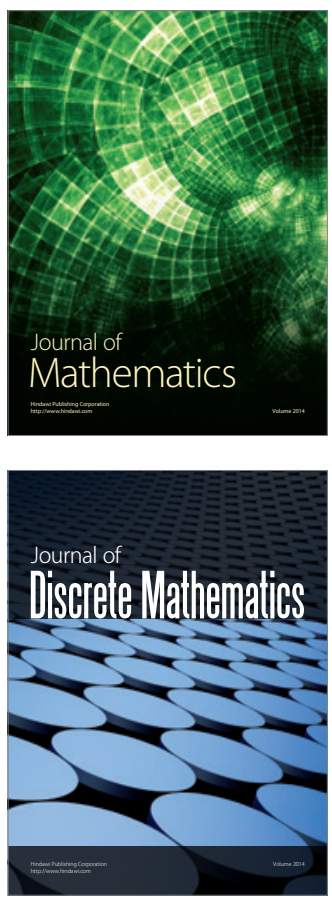

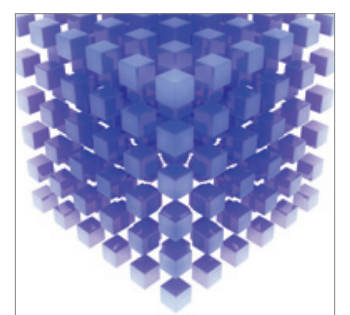

Mathematical Problems in Engineering
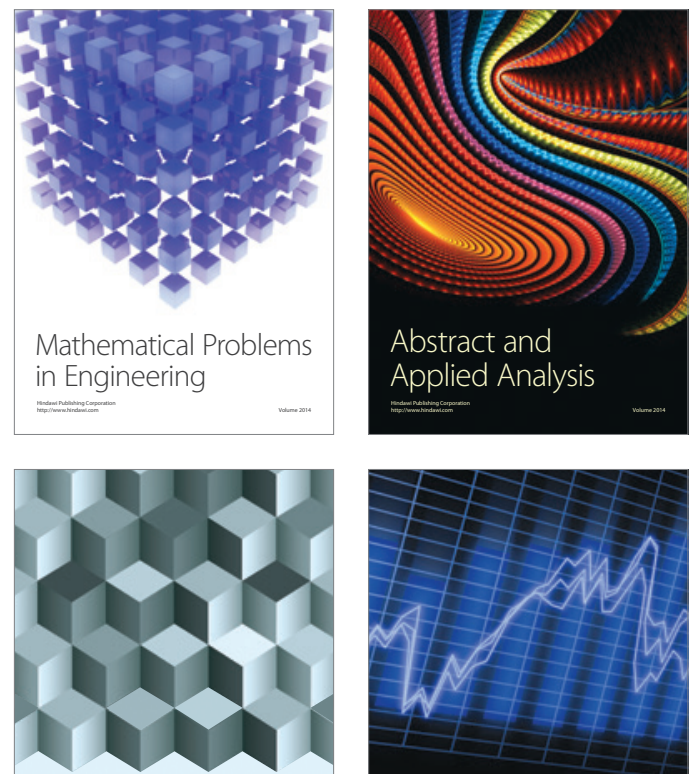

Journal of

Function Spaces

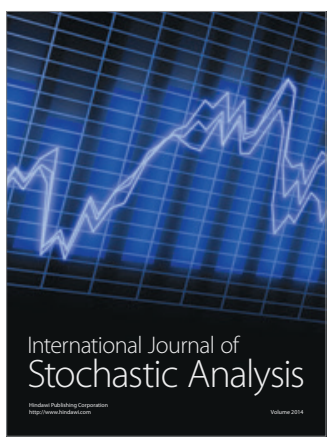

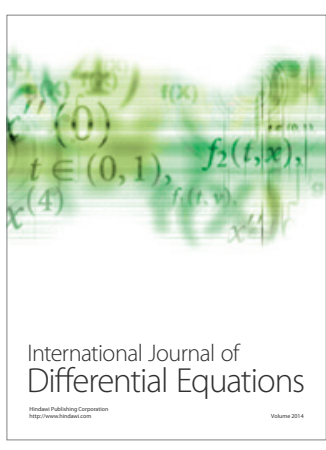
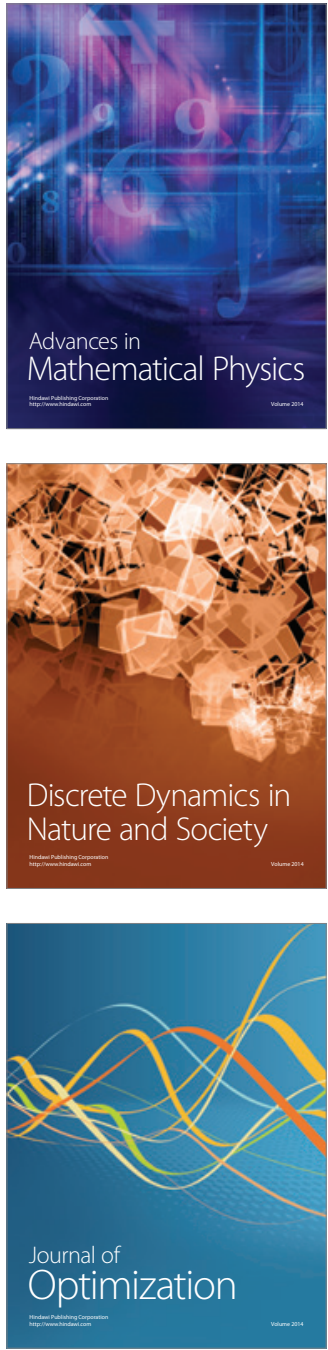